Submitted to The Astronomical Journal

\title{
High-Redshift Quasars Found in Sloan Digital Sky Survey Commissioning Data VI. Sloan Digital Sky Survey Spectrograph Observations \&
}

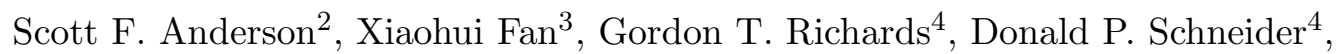 \\ Michael A. Strauss 目, Daniel E. Vanden Berk 6 , James E. Gunn国, Gillian R. Knapp目, \\ David Schlegel迋, Wolfgang Voges \\ Robert Brunner 1, Istvan Csabái10,11, Mamoru Doi 12, Masataka Fukugita 13, B, Željko Ivezió5, \\ Donald Q. Lamb14, Jon Loveday 15 , Robert H. Lupton 1 , Timothy A. McKay 16 , Jeffrey A. Munn17, \\ R.C. Nichol 18 , G.P. Szokoly 19 , and Donald G. York 13 \\ email addresses: anderson@astro.washington.edu, fan@sns.ias.edu, gtr@astro.psu.edu, \\ dps@astro.psu.edu, strauss@astro.princeton.edu,danvb@fnal.gov
}

\footnotetext{
${ }^{1}$ Based on observations obtained with the Sloan Digital Sky Survey and with the Apache Point Observatory 3.5-m telescope, which are owned and operated by the Astrophysical Research Consortium

${ }^{2}$ University of Washington, Department of Astronomy, Box 351580, Seattle, WA 98195.

${ }^{3}$ The Institute for Advanced Study, Princeton, NJ 08540.

${ }^{4}$ Department of Astronomy and Astrophysics, The Pennsylvania State University, University Park, PA 16802.

${ }^{5}$ Princeton University Observatory, Princeton, NJ 08544.

${ }^{6}$ Fermi National Accelerator Laboratory, P.O. Box 500, Batavia, IL 60510.

${ }^{7}$ Max-Planck-Institue für extraterrestrische Physik, Postfach 1603, 85750 Garching, Germany.

${ }^{8}$ Apache Point Observatory, P.O. Box 59, Sunspot, NM 88349-0059.

${ }^{9}$ Astronomy Department, California Institute of Technology, Pasadena, CA 91125.

${ }^{10}$ Department of Physics and Astronomy, Johns Hopkins University, 3701 University Drive, Baltimore, MD 21218.

${ }^{11}$ Department of Physics of Complex Systems, Eötvös University, Pázmáy Péter sétány 1/A, H-1117, Budapest, Hungary.

${ }^{12}$ Department of Astronomy and Research Center for the Early Universe, School of Science, University of Tokyo, Mitaka, Tokyo 181-0015, Japan.

${ }^{13}$ Institute for Cosmic Ray Research, University of Tokyo, Midori, Tanashi, Tokyo 188-8588, Japan

${ }^{14}$ Astronomy and Astrophysics Center, University of Chicago, 5640 South Ellis Avenue, Chicago, IL 60637.

${ }^{15}$ Astronomy Centre, University of Sussex, Falmer, Brighton BN1 9QJ, UK.

${ }^{16}$ Department of Physics,University of Michigan, 500 East University, Ann Arbor, MI 48109.

${ }^{17}$ US Naval Observatory, Flagstaff Station, P.O. Box 1149, Flagstaff, AZ 86002-1149.

${ }^{18}$ Dept. of Physics, Carnegie Mellon University, 5000 Forbes Ave., Pittsburgh, PA 15232.

${ }^{19}$ Astrophysikalisches Institut Potsdam, Germany.
} 


\begin{abstract}
We present results on over 100 high-redshift quasars found in the Sloan Digital Sky Survey (SDSS), using automated selection algorithms applied to SDSS imaging data and with spectroscopic confirmation obtained during routine spectroscopic operations of the Sloan 2.5-m telescope. The SDSS spectra cover the wavelength range $3900-9200 \AA$ at a spectral resolution of 1800, and have been obtained for 116 quasars with redshifts greater than 3.94; 92 of these objects were previously uncataloged, significantly increasing the current tally of published $z>4$ quasars. The paper also reports observations of five additional new $z>4.6$ quasars; all were found from the SDSS imaging survey and spectroscopically confirmed with data from the Apache Point Observatory's 3.5-m telescope. The $i^{\prime}$ magnitudes of the quasars range from 18.03 to 20.56. Of the 97 new objects in this paper, 13 are Broad Absorption Line quasars. Five quasars, including one object at a redshift of 5.11 , have $20 \mathrm{~cm}$ peak flux densities greater than $1 \mathrm{mJy}$. Two of the quasars, both at $z \approx 4.5$, have very weak emission lines; one of these objects is a radio source. Nineteen of the newly-discovered objects have redshifts above 4.6 , and the maximum redshift is $z=5.41$; among objects reported to date, the latter is the third highest redshift AGN, and penultimate in redshift among luminous quasars.
\end{abstract}

Subject headings: cosmology: early universe — quasars:individual

\title{
1. Introduction
}

The past few years have seen a dramatic increase in both the number of known high-redshift quasars and in the highest quasar redshift. Large area surveys, using multicolor selection techniques, have identified a number of quasars at redshifts larger than 5.0, including one object at a redshift of 5.80 (Fan et al. 2000b). Surveys using photographic plates (e.g., Kennefick, Djorgovski, \& de Carvalho 1995b; Storrie-Lombardi et al. 2000, Sharp et al. 2001) and CCDs (the Sloan Digital Sky Survey (SDSS); York et al. 2000) have now produced a data base of well over 100 quasars with redshifts larger than four. Given the current pace of discovery, we expect the number of such quasars will increase by many factors in the near future.

In this series of papers we have already presented SDSS discoveries of more than 100 quasars with redshifts larger than 3.5 (four quasars with redshifts larger than 4.95); all were initially identified in SDSS imaging data and spectroscopic confirmation was obtained using the Apache Point Observatory 3.5-m, Hobby-Eberly, and Keck telescopes (see Schneider et al. 2001 for a summary of the SDSS high-redshift quasars). The SDSS spectrographs began operation in 
early 2000 (see Castander et al. 2001), and in the past year have returned spectra of nearly 9000 quasars; for the initial results of the SDSS quasar survey see Richards et al. (2001b) and Vanden Berk et al. (2001). In this paper we report the first results of the SDSS spectroscopic survey for high-redshift quasars: 116 quasars (92 previously unknown) with redshifts larger than 3.94 identified by commissioning versions of the SDSS Quasar Target Selection Software; the final version of this code is presented in Richards et al. (2001a). In addition, we also describe five new $z>4.6$ quasars that were found in the SDSS imaging data and spectroscopically confirmed with the Apache Point Observatory 3.5-m telescope. Finding charts for all objects lacking published identifications are given in Figure 1.

The SDSS imaging observations and target selection are described in $\S 2$, and the spectroscopic observations of the quasar candidates are presented in $\S 3$. The properties of the quasars are reviewed in $\S 4$, and a brief discussion appears in $\S 5$. Throughout this paper we will adopt the cosmological model with $H_{0}=50 \mathrm{~km} \mathrm{~s}^{-1} \mathrm{Mpc}^{-1}, \Omega_{0}=1.0$, and $\Lambda=0.0$.

\section{Sloan Digital Sky Survey Imaging and Quasar Target Selection}

The Sloan Digital Sky Survey uses a CCD camera (Gunn et al. 1998) on a dedicated 2.5-m telescope (Siegmund et al. 2001) at Apache Point Observatory, New Mexico, to obtain images in five broad optical bands over 10,000 $\mathrm{deg}^{2}$ of the high Galactic latitude sky centered approximately on the North Galactic Pole. The five filters (designated $u^{\prime}, g^{\prime}, r^{\prime}, i^{\prime}$, and $z^{\prime}$ ) cover the entire wavelength range of the CCD response (Fukugita et al. 1996; Fan et al. 2001). Photometric calibration is provided by simultaneous observations with a 20-inch telescope at the same site. The survey data processing software measures the properties of each detected object in the imaging data, and determines and applies both astrometric and photometric calibrations (Pier et al. 2001; Lupton et al. 2001). At the time of this writing (March 2001) substantially more than 1000 sq. deg. have been observed with the SDSS, although some of the data do not meet the strict survey requirements. The finding charts in Figure 1 were made from $i^{\prime}$-band data taken with the SDSS survey camera.

The high photometric accuracy, good image quality, and five wavelength bands covering the optical and near-infrared of the SDSS imaging survey produce an extremely effective data base from which to identify quasars. Since the spectra of quasars and stars differ considerably, multicolor surveys have long been the primary technique employed to optically select quasars (e.g., Schmidt \& Green 1983 and references therein). Fan (1999) calculated the expected location, as a function of redshift, of quasars in SDSS color-space; his results suggested that the SDSS could effectively identify quasars at most redshifts below $\approx 5-6$. Richards et al. (2001b) presented the

SDSS colors of more than 2600 quasars with $0<z<5$; the data show tight color-redshift relations for the vast majority of quasars, and the median relation to a large extent follows the predictions of Fan (1999). 
The presence of the $i^{\prime}$ and $z^{\prime}$ filters in the SDSS camera make it possible to effectively identify high-redshift quasars. The combination of the strong Lyman $\alpha$ emission line, substantial absorption due to the Lyman $\alpha$ forest, and the smooth continuum longward of the Lyman $\alpha$ emission line cause the SDSS colors of most quasars with redshifts above $\approx 3.3$ to be considerably different than SDSS colors of stars (e.g., Fan 1999; Fan et al. 1999; Richards et al. 2001b). As one increases the quasar redshift above $\approx 5.8$, the Lyman $\alpha$ emission line leaves the $i^{\prime}$ band and enters the $z^{\prime}$ filter, so detections of most $z \approx 6$ quasars will rely on only one filter (see Fan 2000b). To date, the SDSS high-redshift quasar selection efficiency (number of quasars divided by the number of quasar candidates) is approximately $60-70 \%$ (e.g., Fan et al. 2001; Schneider et al. 2001) for objects with $i^{*}<20.0$; this is a much higher value than that achieved in previous investigations in this field. The contaminants in the SDSS high-redshift quasar candidates are late-type stars, narrow emission line galaxies at low redshift, and "E+A galaxies" at $z \approx 0.4$ (Fan et al. 1999).

One of the primary goals of the SDSS is to obtain spectra of a sample of $\approx 100,000$ quasars selected from the SDSS imaging data (York et al. 2000). The SDSS Quasar Target Selection Algorithm uses information from the SDSS photometric catalogs (e.g., morphology, magnitude) to produce a list of quasar candidates to be included in the SDSS spectroscopic survey. The details of the selection are given in Richards et al. (2001a). One of the primary tasks of the SDSS commissioning period was to refine the quasar selection technique; during this time a number of versions of the quasar selection algorithm were used.

It is important to note that the objects described in this paper do not constitute a complete sample. Although most of the objects were identified by an automated selection technique, the details of the selection procedure varied from field to field as the effectiveness of the algorithm was tested during the commissioning period.

The planned SDSS complete sample is expected to consist of objects whose colors are distinct from those of stars with $15<i^{\prime}<19.2$ (the bright limit is set to avoid saturation properties in the SDSS spectrographs), objects with the colors of $z>3.0$ quasars with $15<i^{\prime}<20.5$, and point sources with $i^{\prime}<19.2$ that are coincident with radio sources in the FIRST survey (Becker, White, \& Helfand 1995). Low-redshift quasar candidates can have a nonstellar appearance. The expectation is that the quasar survey will have an efficiency (quasars:quasar candidates) of at least $65 \%$ and a completeness of at least $90 \%$ (i.e., $90 \%$ of previously known quasars will be recovered).

\section{Spectroscopy of Quasar Candidates}

The SDSS spectroscopic survey is carried out by two fiber-fed double spectrographs mounted at the Cassegrain focus of the SDSS 2.5-m telescope (see York et al. 2000; Castander et al. 2001; and Uomoto et al. 2001 for details). Each spectrograph contains blue (3900-6200 $\AA$ ) and red (5800-9200 A) beams that produce spectra at a resolution of $\approx 1800$. A total of 320 fibers enter 
each spectrograph; a single observation covers targets located in a $3^{\circ}$ diameter field. The fibers subtend a diameter of $3^{\prime \prime}$ on the sky, and because of mechanical constraints the fibers must be separated by at least $55^{\prime \prime}$. In each field, 32 of the 640 fibers are assigned to measuring the sky, and approximately eight fibers are used for photometric standards and three fibers observe reddening standards. Typically about 100 quasar candidate spectra are taken in a 45-minute observation of a field. For this paper we considered spectroscopic observations of 138 fields, which cover an effective area of approximately 700 sq deg; nearly 9000 quasar spectra have been obtained to date.

The data, along with the associated calibration frames, are processed by the SDSS Spectroscopic Pipeline (Frieman et al. 2001), which removes instrumental effects, extracts the spectra, calculates the wavelength calibration, subtracts the sky spectrum and removes the atmospheric absorption bands, and performs the flux calibration. The spectra are then classified (e.g., star, galaxy, quasar) and redshifts are determined by the pipeline software.

We selected objects whose redshifts, as determined by the SDSS spectroscopic pipeline software, were larger than 3.95, and supplemented this list by visually inspecting the processed spectra and identifying objects whose redshifts appeared to be four or larger. A visual inspection of these candidates produced a set of 116 quasars with redshifts ranging from 3.94 to 5.41 . Although the C IV emission line lies beyond the red cutoff of the spectrograph when the redshift exceeds 4.8 , the quality of the spectra and the distinctive appearance of the Lyman $\alpha+\mathrm{N} \mathrm{V}$ emission line and Lyman $\alpha$ forest region in these objects allows single-line redshifts to be assigned with high confidence. SDSS spectra of 27 of the quasars are displayed in Figure 2.

The high quality of the data is particularly notable given that the spectra, of objects with $i^{*} \approx 20$, were obtained with a 2.5 -m telescope in modest length exposures (less than an hour in clear, good seeing conditions).

An additional five $z>4.6$ quasars, identified as high-redshift candidates in the SDSS imaging data, were spectroscopically confirmed with the Double Imaging Spectrograph on the Apache Point Observatory 3.5-m telescope. The spectra covered the wavelength range 4000-10,000 , with a spectral resolution of $12 \AA$ (blue camera) and $23 \AA$ (red camera); all exposure times were 3600 s. (For details of the instrument configuration see Fan et al. 1999). Redshifts for these five objects were determined using the techniques described in Fan et al. (2001). The spectra of the five quasars are shown in Figure 3.

\section{Discussion}

Table 1 provides basic data (uniform and of high quality) for all 121 quasars. The object name format is SDSSp Jhhmmss.ss+ddmmss.s, where the coordinate equinox is J2000, and the "p" refers to the preliminary nature of the astrometry. The reported magnitudes are based on a preliminary photometric calibration; to indicate this, the filters have an asterisk instead of a prime superscript (e.g., $g^{*}$ rather than $g^{\prime}$ ). The estimated astrometric accuracies in each coordinate 
are $0.10^{\prime \prime}$ and the calibration of the photometric measurements is accurate to 0.04 magnitudes in the $g^{\prime}, r^{\prime}$, and $i^{\prime}$ filters and 0.06 magnitudes in the $u^{\prime}$ and $z^{\prime}$ bands. Throughout the text, object names will frequently be abbreviated as SDSShhmm+ddmm.

Slightly more than $80 \%$ (97) of the quasars in Table 1 were previously unknown, including all five of the $z>4.6$ "APO" objects. Eighteen of the objects had been previously identified in SDSS data; they are included in this study because of the improved photometry and the newly obtained SDSS spectra. Four quasars in Table 1 were discovered by the Automated Plate Measuring facility (APM) survey (see Storrie-Lombardi et al. 2000), and two quasars are Palomar Sky Survey (PSS) objects (Kennefick, Djorgovski, \& de Carvalho 1995b). The final column in Table 1 provides the references for all 24 of the previously known quasars independently recovered here.

The locations of the quasars in SDSS color space are shown in Figure 4. As expected from the predictions of Fan (1999) and demonstrated by previous SDSS high-redshift quasar studies (e.g., see summary in Schneider et al. 2001), the colors of quasars with redshifts between 4.0 and 4.6 are well-separated from the colors of stars in the $\left(g^{*}-r^{*}\right),\left(r^{*}-i^{*}\right)$ diagram (the distance between stars and a typical quasar at this redshift is approximately one magnitude). At redshifts above $\approx 4.6$ the $\left(r^{*}-i^{*}\right),\left(i^{*}-z^{*}\right)$ diagram becomes a very useful aid, because at these redshifts the errors in the $g^{*}-r^{*}$ color can become large due to Lyman-limit systems entering the $g^{*}$ band.

Figure 5 shows the relationship between the $\left(r^{*}-i^{*}\right)$ color and redshift for the 121 quasars in Table 1. Between redshifts of 4.0 and 4.5, the mean color slightly increases, and the colors in this redshift range have a dispersion about the mean of 0.10-0.15 mag. At redshifts above 4.5, when the Lyman $\alpha$ emission line moves from the $r^{\prime}$ to the $i^{\prime}$ filter and the Lyman $\alpha$ forest blankets the $r^{\prime}$ bandpass, the $\left(* r^{*}-i^{*}\right)$ measurements rapidly increase to 1.5 and larger. The large dispersion in the color at $z>4.5$ is likely to arise from the variation in strength of the Lyman $\alpha$ forest along different lines of sight.

The redshift distribution of the quasars in Table 1 is shown in Figure 6 . The numbers decline rapidly with redshift (only about $10 \%$ of the objects have $z>4.8$ ), and there is a slight dip near $z=4.5$ due to the difficulty of separating quasars of this redshift from the stellar locus (see Fan et al. 2001) using the early commissioning selection algorithm. Figure 7 displays the distribution of $i^{*}$ magnitudes of the quasars. The bulk of the objects have $19.2<i^{*}<20.4$; the brightest object has $i^{*}=18.03$ and the faintest quasar has $i^{*}=20.56$. Fourteen of the quasars have $i^{*}<19.0$.

These objects are moderately luminous quasars; 3C 273, which has $M_{B}=-27.0$ in our adopted cosmology, would appear in our sample $\left(i^{*}<20.6\right)$ out to a redshift of approximately 4.8. The most luminous objects in Table 1 are over two magnitudes brighter than 3C 273.

The sample includes nineteen new quasars with $z>4.6$, including three new quasars with $z>5$ : SDSS0231-0728, SDSS0756+4104, and SDSS0913+5919.

The sample contains one set of quasars that are relatively close together on the sky: $\operatorname{SDSS1108-0059}(z=4.01)$ and $\operatorname{SDSS1108-0058}(z=4.56)$ are separated by only $80^{\prime \prime}$. The 
projected comoving separation of the lines of sight at $z=4.01$ is approximately $500 \mathrm{kpc}$.

Sixteen of the 121 quasars, and 13 of the 97 newly discovered ones, are either certain or probable BAL quasars; this is consistent with the $10 \%$ BAL fraction found in optically selected quasars at lower redshift (Weymann et al. 1991). The spectra of many of the quasars have narrow absorption line systems, both intervening and intrinsic.

Five quasars in Table 1 have peak $20 \mathrm{~cm}$ (FIRST) flux densities greater than $2.5 \mathrm{mJy}$. Two objects which are not included in the FIRST survey area were detected by the NRAO VLA Sky Survey (NVSS, Condon et al. 1998; see notes on individual objects). An additional two quasars were detected by FIRST, but were fainter than than the NVSS limit. Four of the radio quasars were previously known; only five of the 97 newly discovered quasars are radio sources above the $\approx 1$ mJy level. One of five quasars, SDSS0913+5919 $(z=5.11)$, is a luminous radio source $(18.1 \mathrm{mJy}$ at $20 \mathrm{~cm})$, and is the most distant known radio-loud quasar. This fraction of radio detections $(\leq 10 \%)$ is comparable to the results found in previous studies of high-redshift quasars (e.g., Schmidt et al. 1995b, Stern et al. 2000a).

None of the quasars are found in the ROSAT All-Sky Survey (RASS) Bright Source Catalog (Voges et al. 1999; Schwope et al. 2000), which has a flux limit of $2.4 \times 10^{-12} \mathrm{erg} \mathrm{cm}^{-2} \mathrm{~s}^{-1}$ in the 0.5 to $2.0 \mathrm{keV}$ band. This result is not unexpected given that with the exception of a few unusual sources (e.g., blazars), high-redshift quasars have X-ray fluxes well below the catalog limit (Kaspi, Brandt, \& Schneider 2000). One quasar, SDSS1737+5828, may have been detected in the RASS Faint Source Catalog (Voges et al. 2000), but the optical/X-ray match is far from certain (see notes on individual objects).

\section{Notes on Individual Objects}

SDSSp J003126.80+150739.6 $(z=4.20)$ : This radio-loud quasar has a flux density of $42.2 \mathrm{mJy}$ at $20 \mathrm{~cm}$ from the NVSS.

SDSSp J012004.83+141108.3 $(z=4.71)$ : This object contains deep C IV and Si IV BAL features, and there may be strong Lyman $\beta+\mathrm{O}$ VI emission. The spectrum was taken with the APO 3.5-m telescope; the data for this object are displayed in Figure 3.

SDSSp J015032.87+143425.6 $(z=4.14)$ : The spectrum of this quasar shows very deep, very broad Si IV and C IV absorption troughs, and also has a narrow, associated absorption line system.

SDSSp J015642.11+141944.4 $(z=4.30)$ : Strong BAL features are apparent in this spectrum, and lines from a given ion have multiple, broad components. 
SDSSp J023137.65-072854.5 $(z=5.41)$ : This object has the highest redshift in our current sample, and is the AGN with the third largest known redshift (following the luminous SDSS quasar with $z=5.80$ found by Fan et al. 2000b, and a low luminosity AGN at $z=5.50$ found by Stern et al. 2000b). While the redshift relies especially on the strong line at $\lambda=7800 \AA$, the redshift is not in doubt given the characteristic line profile and continuum discontinuity, plus the presence of a Lyman limit system at a rest wavelength of $910 \AA$. There is also strong evidence for Lyman $\beta+\mathrm{O}$ VI and N V emission at a consistent redshift. The absorption just shortward of the Lyman $\alpha$ emission line is exceptionally strong; the flux appears to drop to zero. This object was observed twice with the SDSS spectrographs, and the two spectra both indicate a redshift of 5.41. SDSSp J023923.47-080105.1 $(z=4.00)$ : This possible BAL quasar appears to have shallow, high-velocity C IV absorption and exceptionally strong O IV+Lyman $\beta$ emission.

SDSSp J024447.79-081606.1 $(z=4.03)$ : At $i^{*}=18.03$, this quasar is both the brightest and the most luminous $\left(M_{B} \approx-29.3\right.$, assuming an ultraviolet-optical spectral index of -0.5$)$ object in the sample.

SDSSp J024457.19-010809.9 $(z=3.96)$ : This bright $\left(i^{*}=18.33\right)$ BAL quasar is an unpublished PSS object.

SDSSp J025204.29+003136.9 $(z=4.10)$ : The spectrum of this object displays deep, broad C IV absorption and strong, multi-component Si IV absorption.

SDSSp J025647.06-085041.4 $(z=4.21)$ : This is a BAL quasar with weak absorption features, the most prominent of which is probably due to $\mathrm{N}$ V.

SDSSp J033005.32-053709.0 $(z=4.09)$ : The spectrum of this BAL quasar shows two narrow C IV features superposed on a broad C IV absorption trough.

SDSSp J034946.61-065730.3 $(z=3.95)$ : The spectrum of this BAL quasar has broad, shallow Si IV and C IV absorption features.

SDSSp J075618.4+410408.6 $(z=5.09)$ : This quasar has the fourth largest redshift discovered by the SDSS.

SDSSp J083946.22+511202.8 $(z=4.39)$ : The FIRST survey measurement of a $20 \mathrm{~cm}$ peak flux density of $40.50 \mathrm{mJy}$ indicates that this is a radio-loud quasar.

SDSSp J085210.89+535949.0 $(z=4.20)$ : This is a BAL quasar with a deep C IV absorption feature.

SDSSp J085634.93+525206.4 $(z=4.72)$ : This object is a BAL quasar; it has a series of broad, deep absorption features. The precise redshift is uncertain given the nature of the BAL features.

SDSSp J091316.56+591921.5 $(z=5.11)$ : This quasar has the second largest redshift in our sample, and is near the faint limit of the SDSS spectroscopic survey. The spectrum (see Figure 2) is of modest signal-to-noise ratio, and there is only one significant emission feature, but the spectral region centered on $\approx 7500 \AA$ displays all the characteristics of Lyman $\alpha$ emission line at $z=5.11$. The radio flux from the object is $18.1 \mathrm{mJy}$ (NVSS); this is a powerful radio quasar.

SDSSp J102043.83+000105.8 $(z=4.30)$ : This quasar was detected by the FIRST survey at a peak flux density of $1.68 \mathrm{mJy}$ at $20 \mathrm{~cm}$. 
SDSSp J110826.32+003706.8 $(z=4.41)$ : This is a BAL quasar with strong, smooth Si IV and C IV absorption features.

SDSSp J123937.18+674020.8 $(z=4.40)$ : The spectrum of this object displays C IV absorption and what may be an $\mathrm{N} \mathrm{V}$ absorption feature; we tentatively classify this as a BAL quasar.

SDSSp J130216.13+003032.1 $(z=4.50)$ : This object has very weak emission lines; the spectrum appears quite similar to the $z=4.62$ object reported by Fan et al. (1999).

SDSSp J141332.36-004909.7 $(z=4.14)$ : It is not clear whether this object is a bona fide BAL or if the C IV and Si IV absorption features are formed from narrow multi-component "associated" absorption. The Lyman $\beta+\mathrm{O}$ VI emission line is exceptionally strong.

SDSSp J144231.73+011055.3 $(z=4.56)$ : This quasar was detected by the FIRST survey at a peak flux density of $1.07 \mathrm{mJy}$ at $20 \mathrm{~cm}$. The spectrum is nearly bereft of emission lines, similar to the $z=4.62$ object reported by Fan et al. (1999); also compare to SDSS1302+0030 noted above.

SDSSp J160501.21-011220.6 $(z=4.92)$ : This quasar was discovered by Fan et al. (2000a); it is the highest redshift BAL yet published, and has the by far largest value of $r^{*}-i^{*}(2.37 \pm 0.15)$ in our sample.

SDSSp J162048.74+002005.7 $(z=4.09)$ : The spectrum of this quasar displays a wealth of absorption phenomena: strong, broad C IV, multi-component Si IV, perhaps Lyman $\alpha+\mathrm{N}$ V, and strong, broad O IV.

SDSSp J173744.87+582829.5 $(z=4.94)$ : This quasar is possibly X-ray detected in the RASS Faint Source Catalog (1RXS J173739.9+582823) with an unabsorbed flux in the $0.1-2.4 \mathrm{keV}$ band of $7.3 \times 10^{-14} \mathrm{erg} \mathrm{s}^{-1} \mathrm{~cm}^{-2}$. The centroid of this X-ray source ( $\approx 7$ photons) is $40^{\prime \prime}$ from the SDSS position, and the chance of at least one unrelated RASS X-ray source superposition in our sample of over one hundred quasars is non-negligible (about 25\%). A higher spatial resolution X-ray image (e.g., from Chandra) would clarify the identification. If confirmed, SDSS1737+5828 would be the highest redshift X-ray source yet detected in the RASS.

\section{Summary}

Calculations based on the measured high-redshift luminosity function (e.g., Schmidt, Schneider \& Gunn 1995a; Kennefick, Djorgovski \& de Carvalho 1995a) and the expected performance of the SDSS camera predicted that the SDSS should produce a sample of well over a thousand quasars at redshifts above four (e.g., Schneider 1999), and that the SDSS automated selection algorithm could identify quasars as distant as a redshift of six. The early results, presented in this paper, are promising. Although less than $10 \%$ of the survey area has been observed spectroscopically, and much of the work to date has been spent fine-tuning both the equipment and the software, the SDSS has already, in "survey mode", selected more than 100 such objects. We are particularly encouraged by the discovery of the quasar at redshift 5.41 ; this object, and the other $z \approx 5.0$ quasars in this paper, show that such objects can be found in an automated manner. 
The SDSS commissioning was completed in Fall 2000, and the survey has now officially commenced. The quasars in this paper do not constitute a complete sample because they were identified with a variety of selection criteria during the commissioning period. We expect that in each year of operation the SDSS will discover 150-200 quasars will redshifts larger than four, and five to ten quasars with redshifts larger than five, all found with well-defined, uniform selection criteria.

This work was supported in part by National Science Foundation grants PHY00-70928 (XF), AST99-00703 (GTR and DPS), and AST00-71091 (MAS). XF and MAS acknowledge additional support from the Princeton University Research Board, and a Porter O. Jacobus Fellowship, and XF acknowledges support from a Frank and Peggy Taplin Fellowship.

The Sloan Digital Sky Survey 20 (SDSS) is a joint project of The University of Chicago, Fermilab, the Institute for Advanced Study, the Japan Participation Group, The Johns Hopkins University, the Max-Planck-Institute for Astronomy (MPIA), the Max-Planck-Institute for Astrophysics (MPA), New Mexico State University, Princeton University, the United States Naval Observatory, and the University of Washington. Apache Point Observatory, site of the SDSS telescopes, is operated by the Astrophysical Research Consortium (ARC). Funding for the project has been provided by the Alfred P. Sloan Foundation, the SDSS member institutions, the National Aeronautics and Space Administration, the National Science Foundation, the U.S. Department of Energy, Monbusho, and the Max Planck Society.

\footnotetext{
${ }^{20}$ The SDSS Web site is http://www.sdss.org/.
} 


\section{REFERENCES}

Becker, R.H., White, R.L., \& Helfand, D.J. 1995, ApJ, 450, 559

Castander, F.J., Nichol, R.C., et al. 2001, AJ, 121, in press

Condon, J.J., Cotton, W.D., Greisen, E.W., Yin, Q.F., Perley, R.A., Taylor, G.B., and Broderick, J.J. 1998, AJ, 115, 1693

Fan, X. 1999, AJ, 117, 2528

Fan, X., Strauss, M.A., Gunn, J.E., Lupton, R.H., et al. 1999, ApJL, 526, 57

Fan, X., Strauss, M.A., Richards, G.T., Newman, J.A., et al. 2001, AJ, 121, 31

Fan, X., Strauss, M.A., Schneider, D.P., Gunn, J.E., Lupton, R.H., Anderson, S.F., et al. 2000a, AJ, 119, 1

Fan, X., Strauss, M.A., Schneider, D.P., Gunn, J.E., Lupton, R.H., Yanny, B., et al. 1999, AJ, 118,1

Fan, X., White, R.L., Davis, M., Becker, R.H., et al. 2000b, AJ, 120, 1167

Frieman, J.A., et al. 2001, in preparation

Fukugita, M., Ichikawa, T., Gunn, J.E., Doi, M., Shimasaku, K., \& Schneider, D.P. 1996, AJ, 111, 1748

Gunn, J.E., Carr, M.A., Rockosi, C.M., Sekiguchi, M., et al. 1998, AJ, 116, 3040

Kaspi, S., Brandt, W.N., \& Schneider, D.P. 2000, AJ, 119, 2031

Kennefick, J., de Carvalho, R.R., Djorgovski, S.G., Wilber, M.M., Dickson, E.S., Weir, N., Fayyad, U., \& Roden, J. 1995a, AJ, 110, 78

Kennefick, J., Djorgovski, S.G., \& de Carvalho, R.R. 1995b, AJ, 110, 2553

Lupton, R.H., Gunn, J.E., \& Szalay, A. 1999, AJ, 118, 1406

Lupton, R.H., Ivezić, Z., et al. 2001, in preparation

Pier, J.R., et al. 2001, in preparation

Richards, G.T., Fan, X., Newberg, H.J., et al. 2001a, in preparation

Richards, G.T., Fan, X., Schneider, D.P., Vanden Berk, D.E., Strauss, M.A., et al. 2001b, AJ, 121, in press

Schlegel, D.J., Finkbeiner, D.P., \& Davis, M. 1998, ApJ, 500, 525 
Schmidt, M., \& Green, R.F. 1983, ApJ, 269, 352

Schmidt, M., Schneider, D.P., \& Gunn, J.E. 1995a, AJ, 110, 68

Schmidt, M., van Gorkom, J.H., Schneider, D.P., \& Gunn, J.E. 1995b, AJ, 109, 473

Schneider, D.P., Fan, X., Strauss, M.A., Gunn, J.E., et al. 2001, AJ, 121, in press

Schwope, A., Hasinger, G., Lehmann, I., Schwarz, R., Brunner, H., Neizvestny, S., Urgryumov, A., Balega, Y., Trümper, J., \& Voges, W., 2000, AN, 321, 1

Sharp, R.G., McMahon, R.G., Irwin, J.J., \& Hodgkin, S.T. 2001, MNRAS, submitted/astroph/0103079

Siegmund, W., et al. 2001, in preparation

Smith, J.D., Djorgovski, S.D., Thompson, D., Brisken, W.F., Neugebauer, G., Matthews, K., Meylan, G., Piotto, G., \& Suntzeff, N.B. 1994a, AJ, 108, 1147

Smith, J.D., Thompson, D., \& Djorgovski, S.D. 1994b, AJ, 107, 24

Stern, D., Djorgovski, S.G., Perley, R.A., de Carvalho, R.R., \& Wall, J.V. 2000a, AJ, 119, 1526

Stern, D., Spinrad, HJ., Eisenhardt, P., Bunker, A.J., Dawson, S., Stanford, S., \& Elston, R. 2000, ApJ, 533, L75

Storrie-Lombardi, L.J., Irwin, M.J., McMahon, R.G., \& Hook, I.M. 2000, MNRAS, in press/astro-ph/0012446

Uomoto, A., et al. 2001, in preparation

Vanden Berk, D.E., Richards, G.T., Bauer, A., et al. 2001, AJ, submitted

Voges, W., et al. 1999, A \& A, 349, 389

Voges, W., et al. 2000, IAUC, 7432

York, D.G., Adelman, J., Anderson, J.E., Anderson, S.F., et al. 2000, AJ, 120, 1579

Weymann, R.J., Morris, S.L., Foltz, C.B., \& Hewett, P.C. 1991, ApJ, 373, 23 


\section{Figure Captions}

Fig. 1.- $(a-d)$ Finding charts for the 98 quasars in this paper that lack published identifications; each individual chart is $100^{\prime \prime}$ on a side. All frames are $i^{\prime}$ images taken with the SDSS camera. The small arrow in the lower left of each chart indicates the direction of north; all charts have "sky" parity, so east is located $90^{\circ}$ counterclockwise from north.

Fig. 2.- (a-i) Spectra of 27 of the quasars taken with the SDSS spectrographs. The spectral resolution is approximately 1800; data points shortward of $4250 \AA$ are not displayed as they have very low signal-to-noise ratio. Displayed are all the $z>4.8$ objects, BALs, and the two $z \approx 4.5$ quasars with extremely weak emission lines.

Fig. 3.- Apache Point Observatory Spectra of the five $z>4.6$ quasars taken with the Double Imaging Spectrograph on the Apache Point Observatory's 3.5-m telescope. The spectral resolution is approximately $23 \AA$, and the exposure times are all $3600 \mathrm{~s}$. Only data from the red beam are displayed as these objects produce little signal below $5500 \AA$.

Fig. 4.- Locations of the 121 quasars in the $\left(g^{*}-r^{*}\right),\left(r^{*}-i^{*}\right)$ (left panel) and $\left(r^{*}-i^{*}\right),\left(i^{*}-z^{*}\right)$ (right panel) color-color diagrams. The quasars are coded by redshift; triangles represent redshifts less than 4.6 and circles are $z>4.6$ quasars. The points and contours represent the colors of 10,000 stars.

Fig. 5.- The $\left(r^{*}-i^{*}\right)$ color of the 121 quasars as a function of redshift. Note the sudden reddening at $z \approx 4.5$ as the Lyman $\alpha$ emission line enters the $i^{\prime}$ band.

Fig. 6.- The redshift distribution of the 121 quasars. Note the steep decline as one moves to larger redshifts. The dip at $z \approx 4.5$ is due to a drop in the detection efficiency at these redshifts of the quasar selection algorithm used during SDSS commissioning (see Fan et al. 2001).

Fig. 7. - The distribution of the $i^{*}$ magnitudes of the 121 quasars. The sharp cutoff near $i * \approx 20.5$ is due to the brightness limit of the SDSS spectroscopic survey. Fourteen of the objects have $i^{*}<19$. 


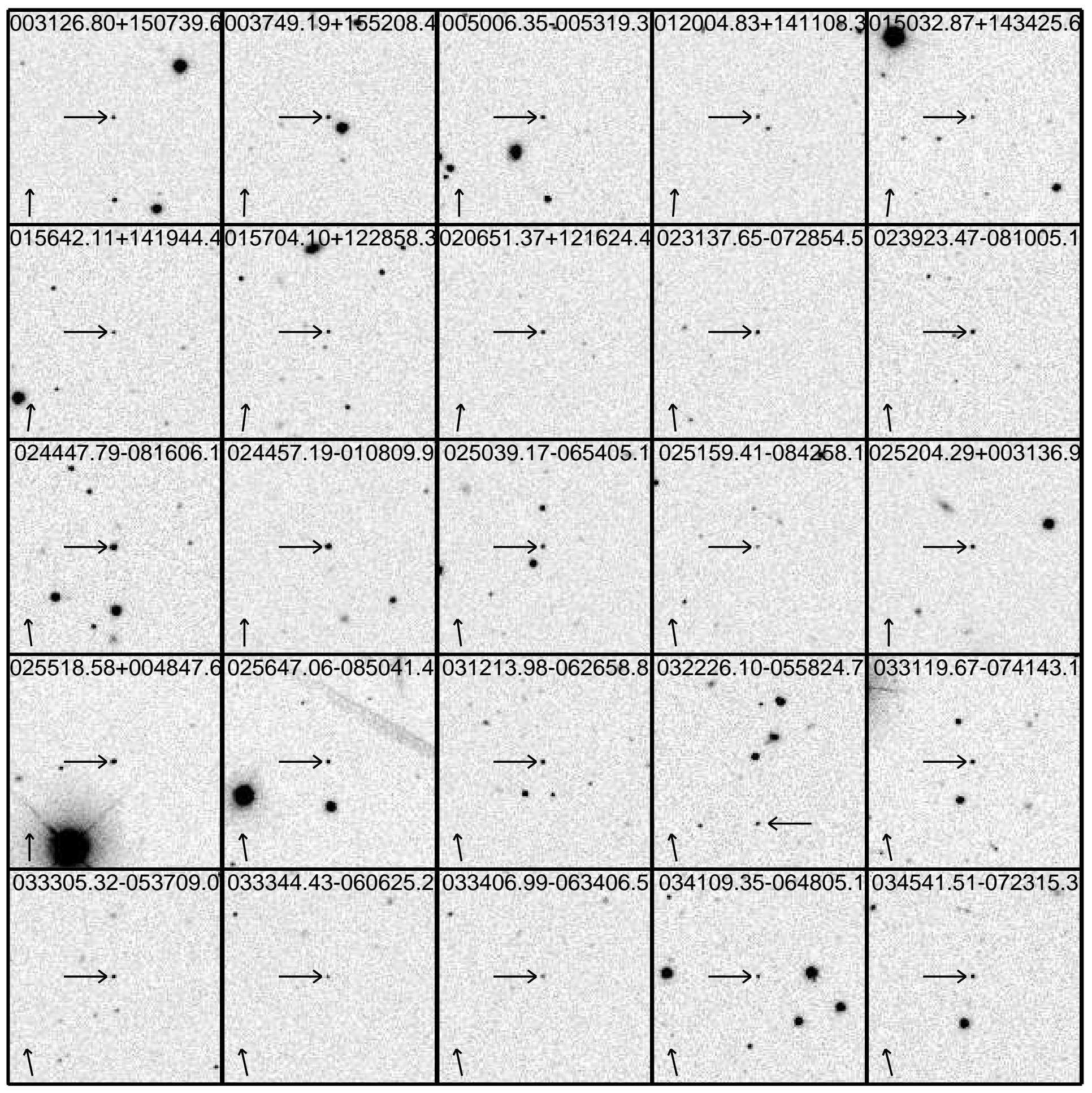




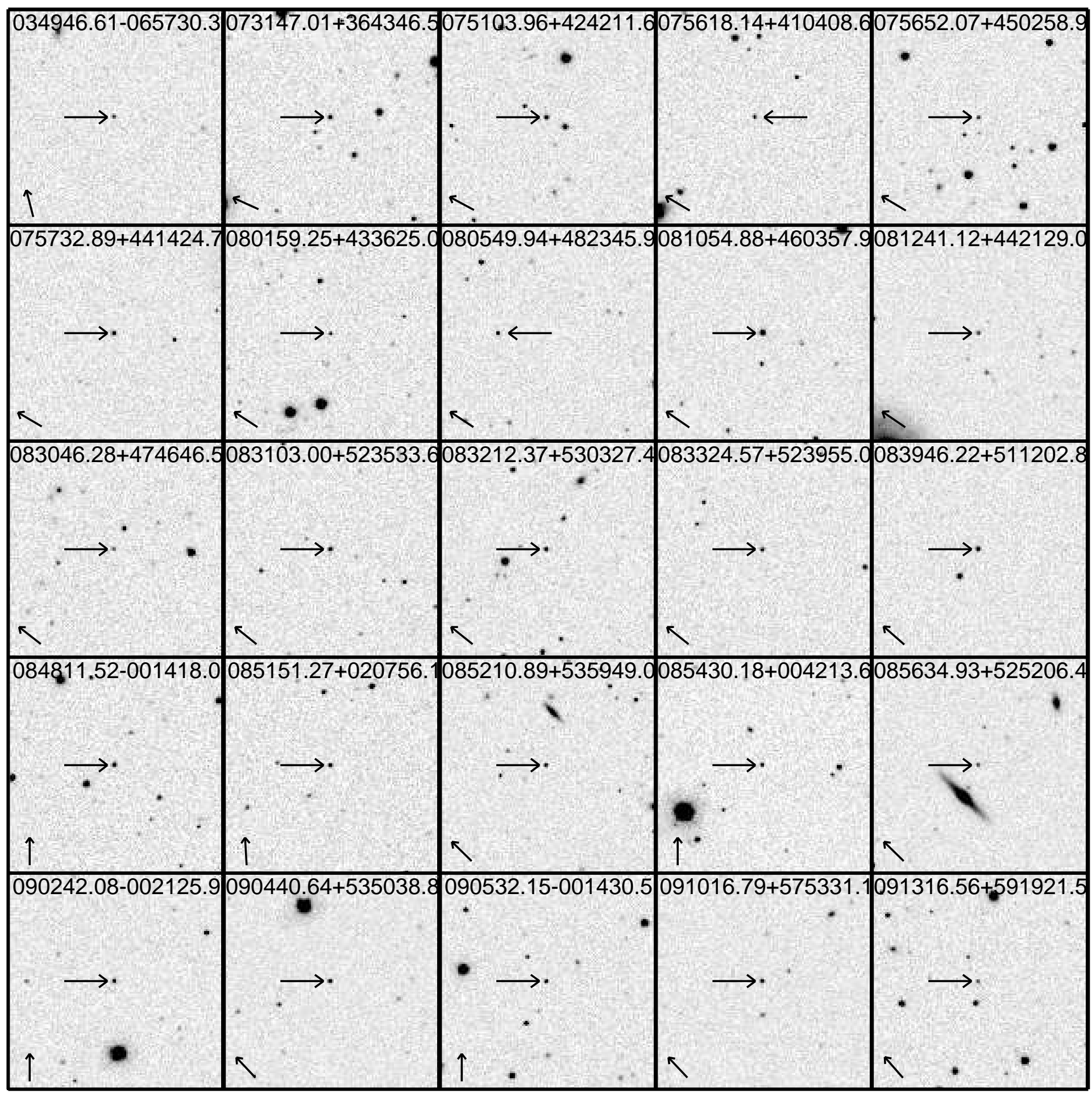




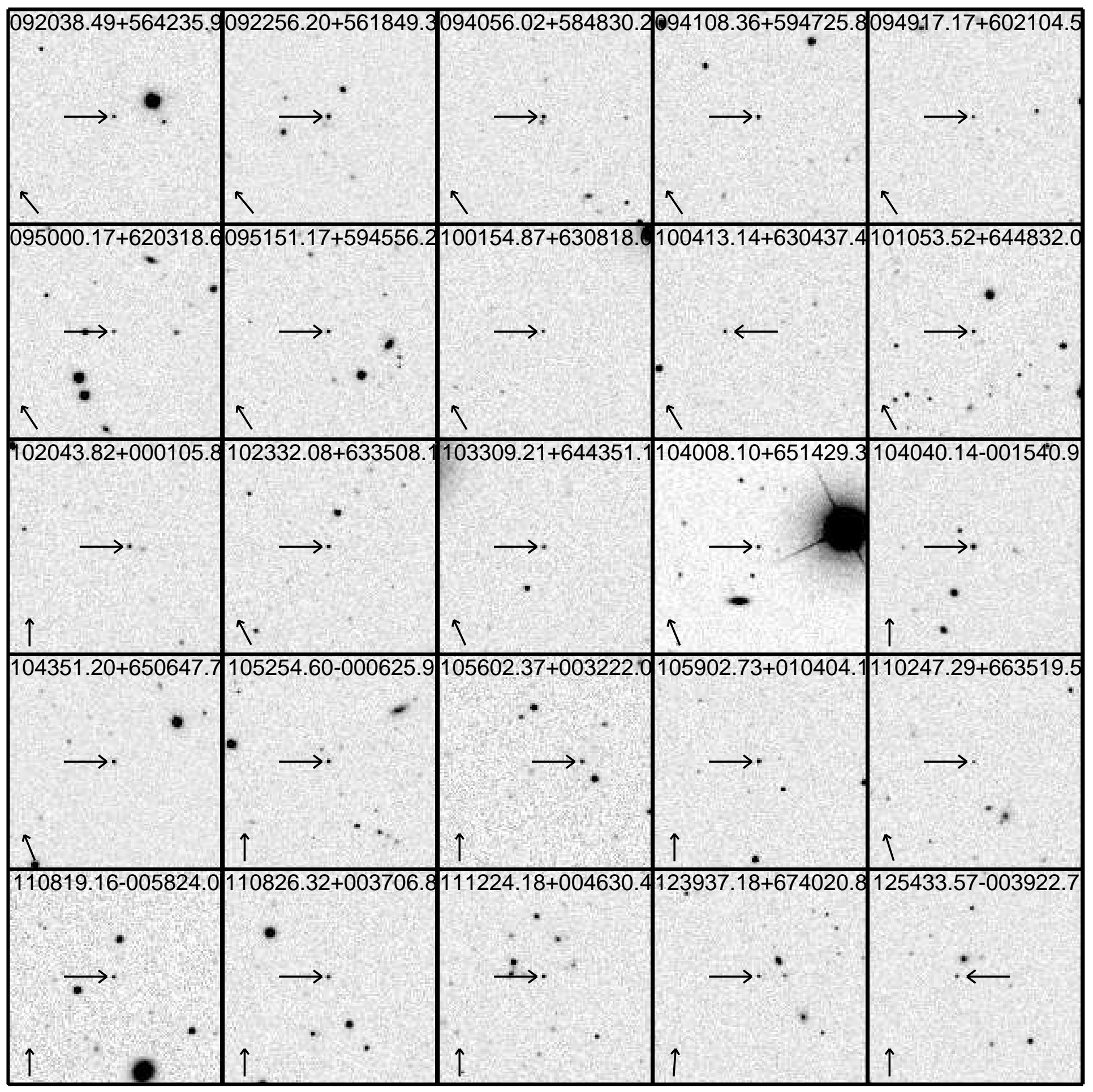




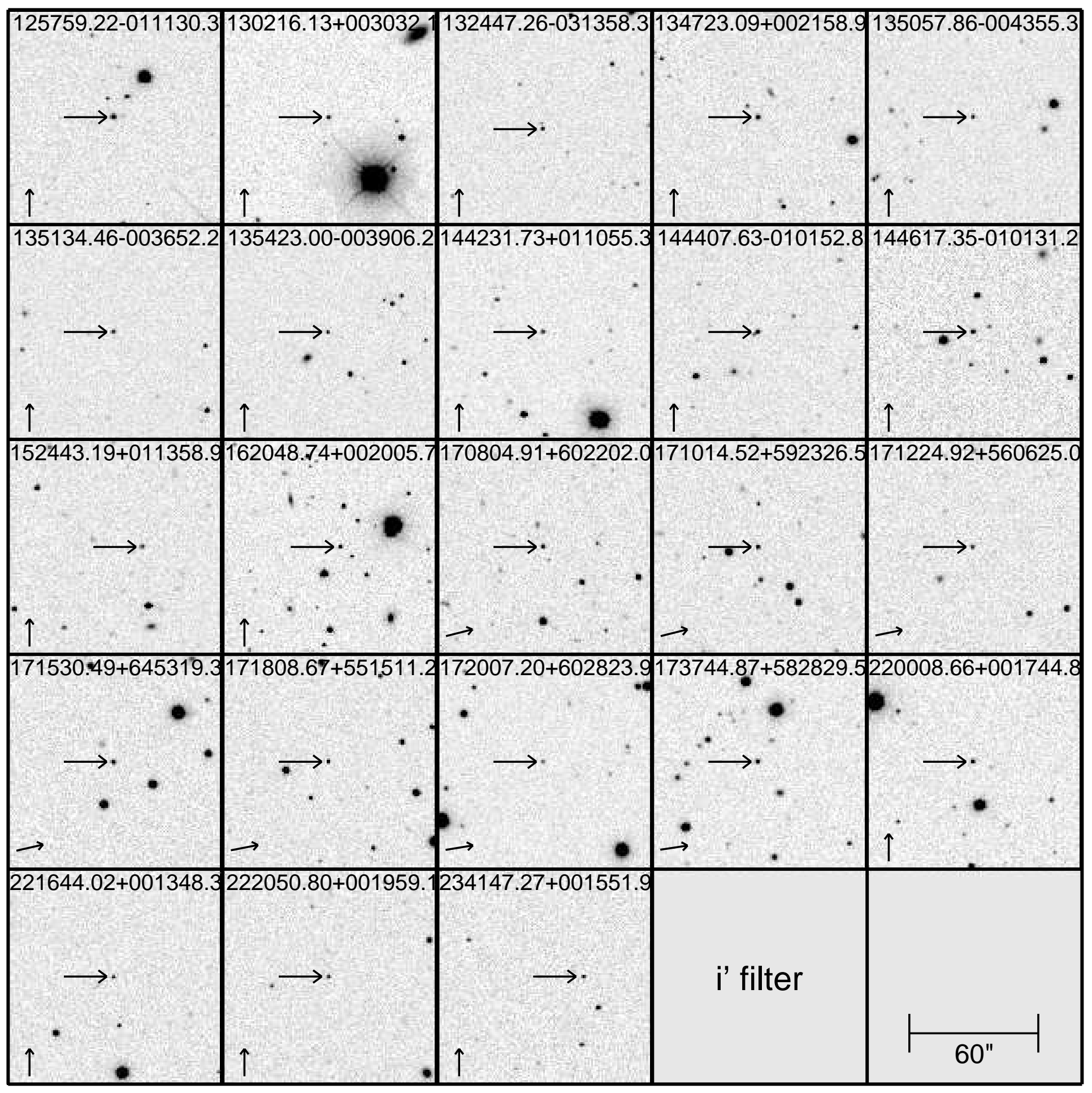




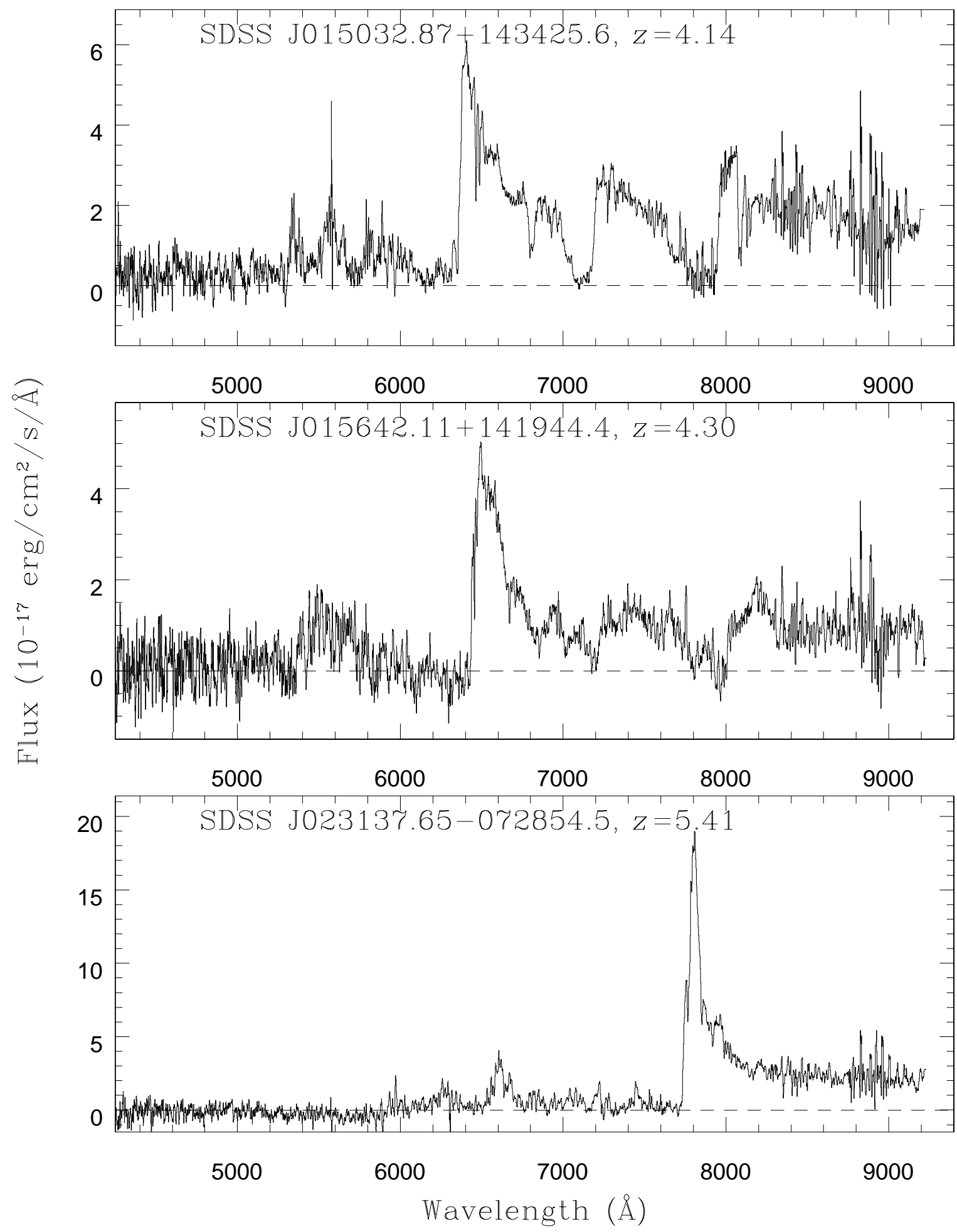




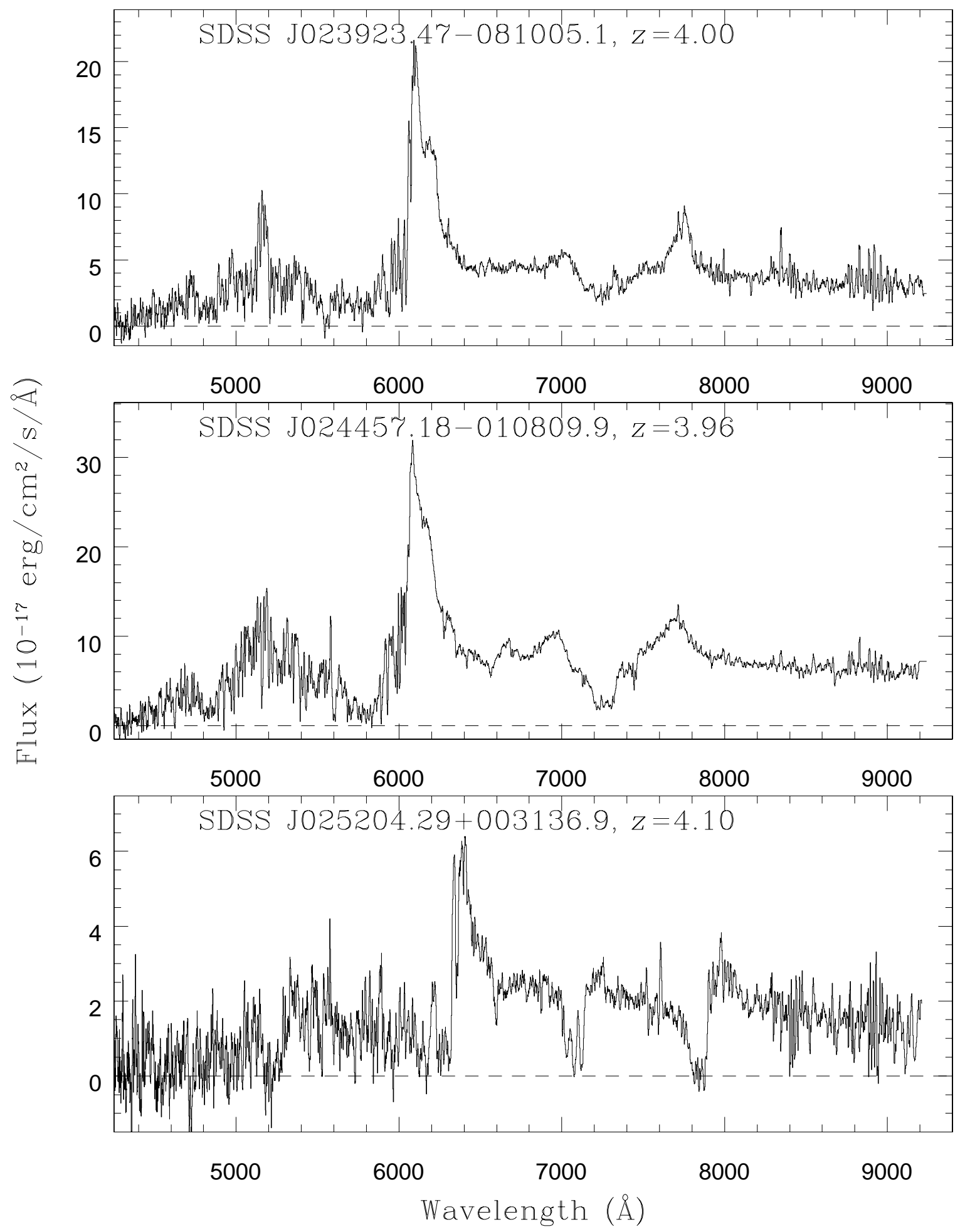




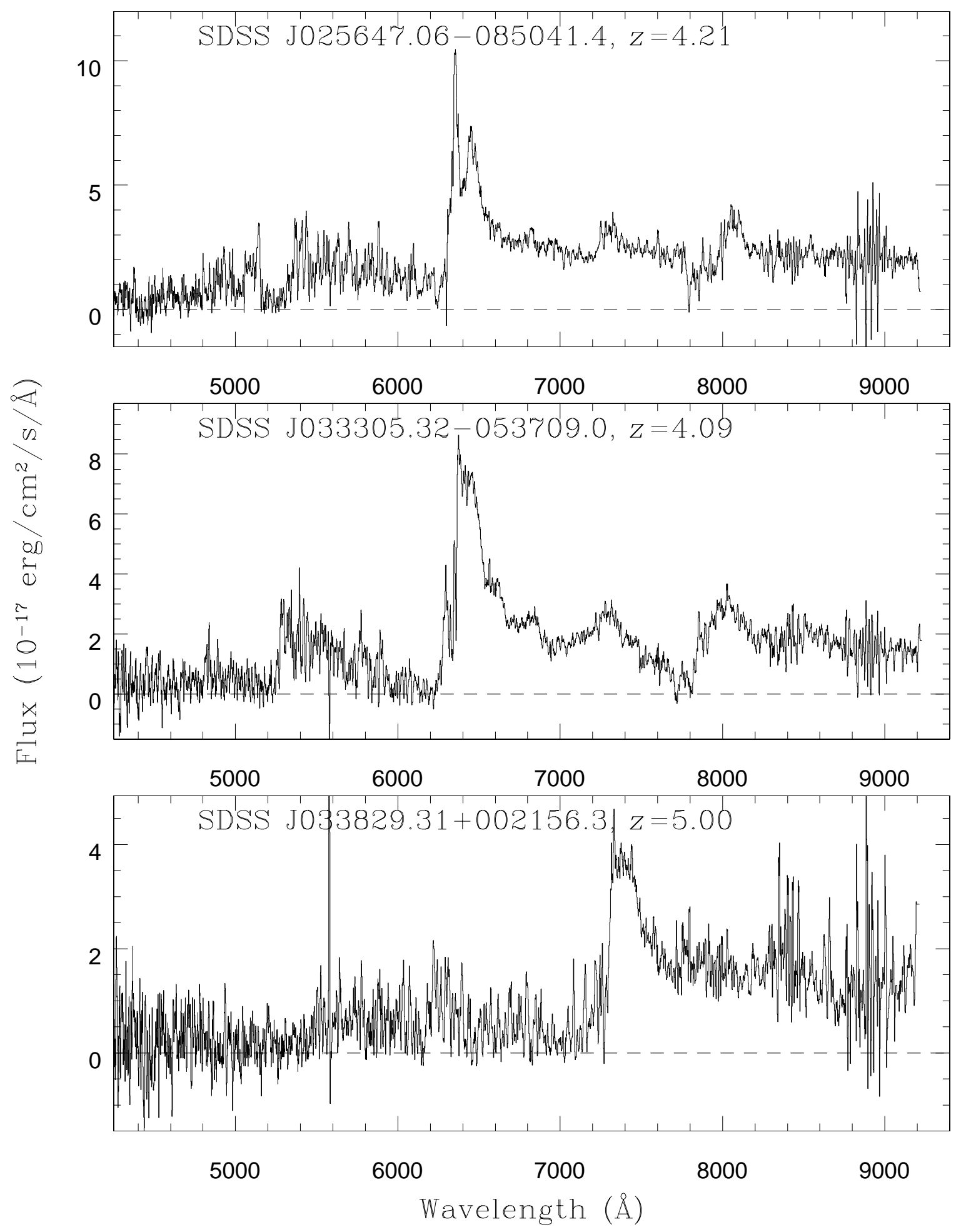




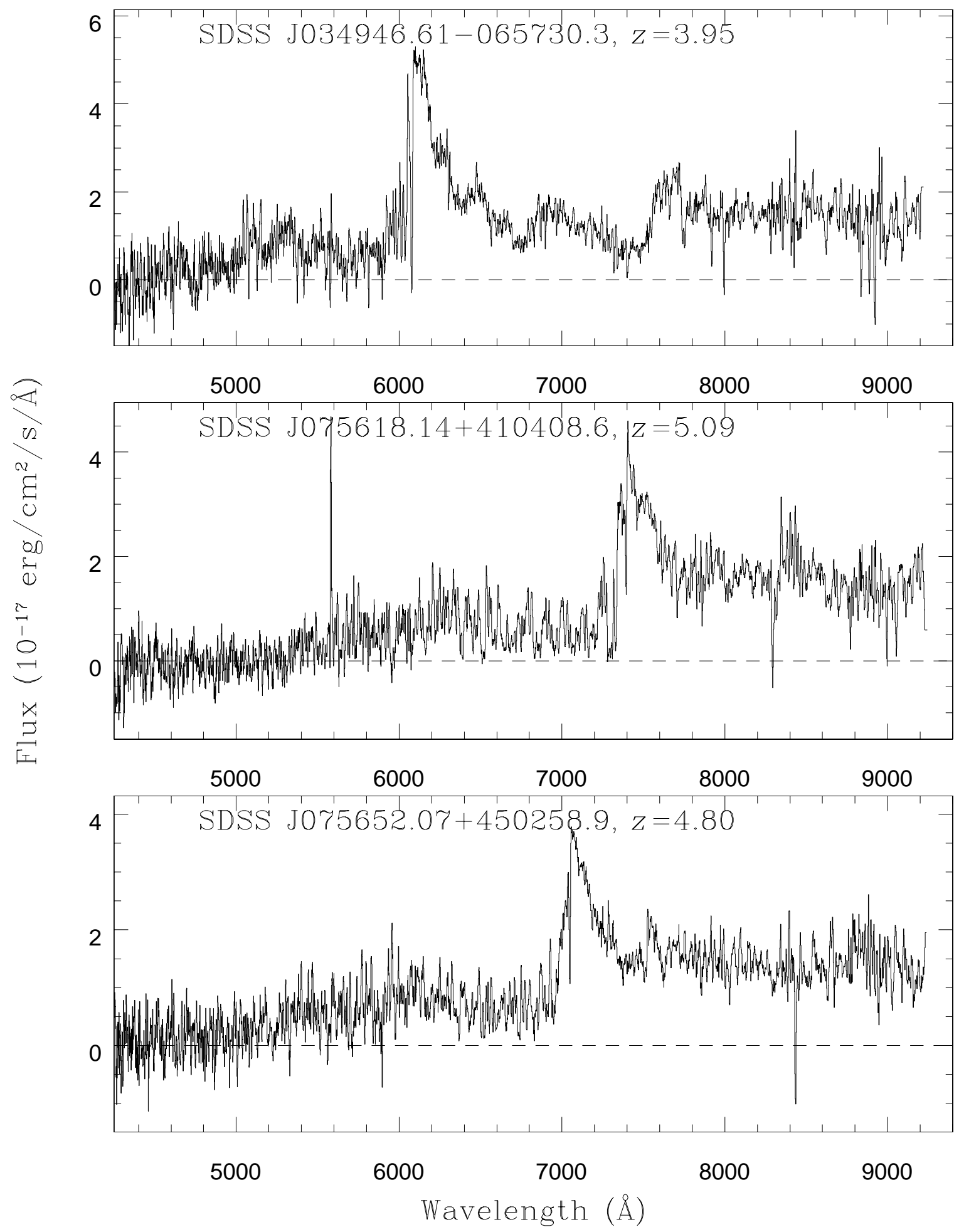




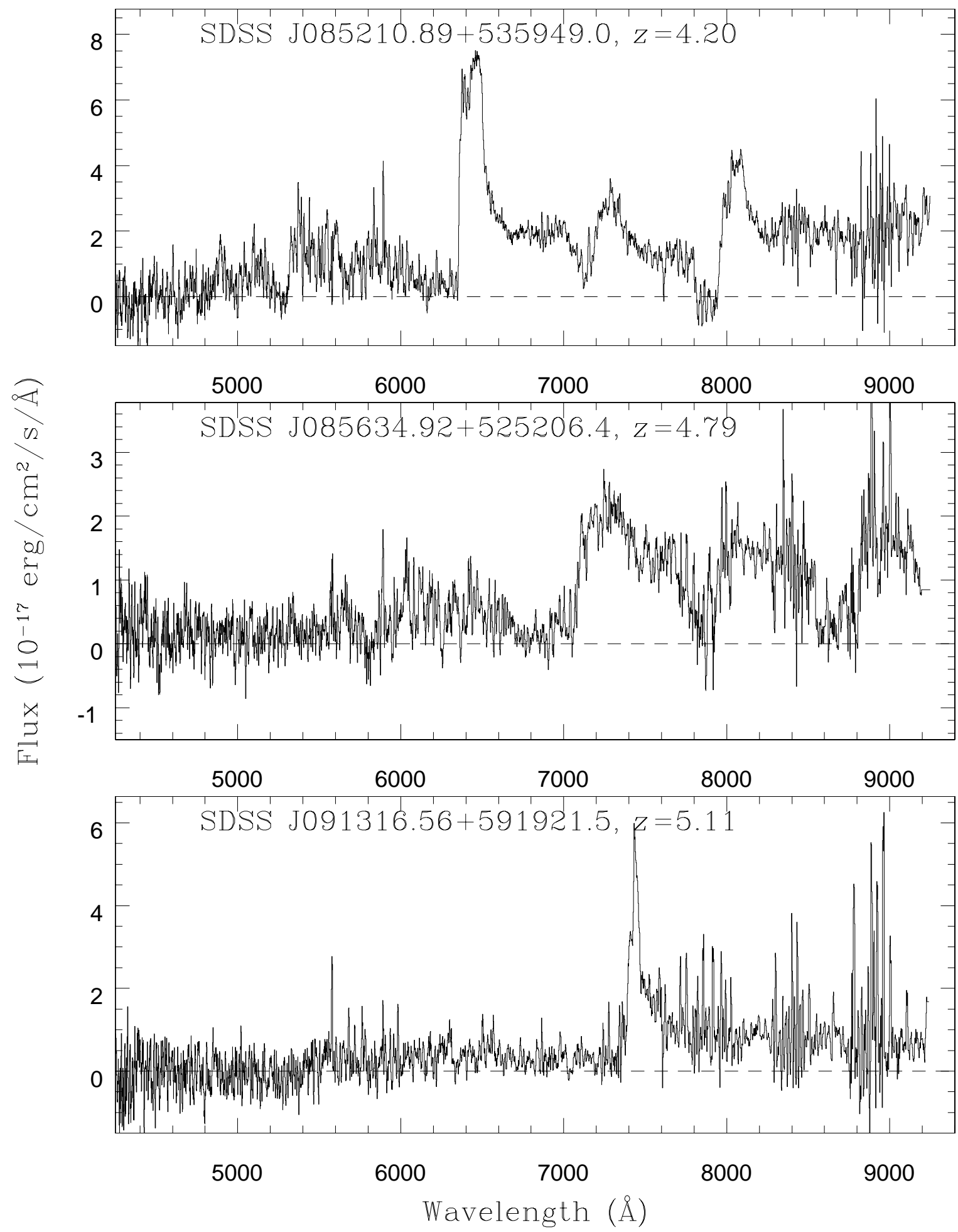




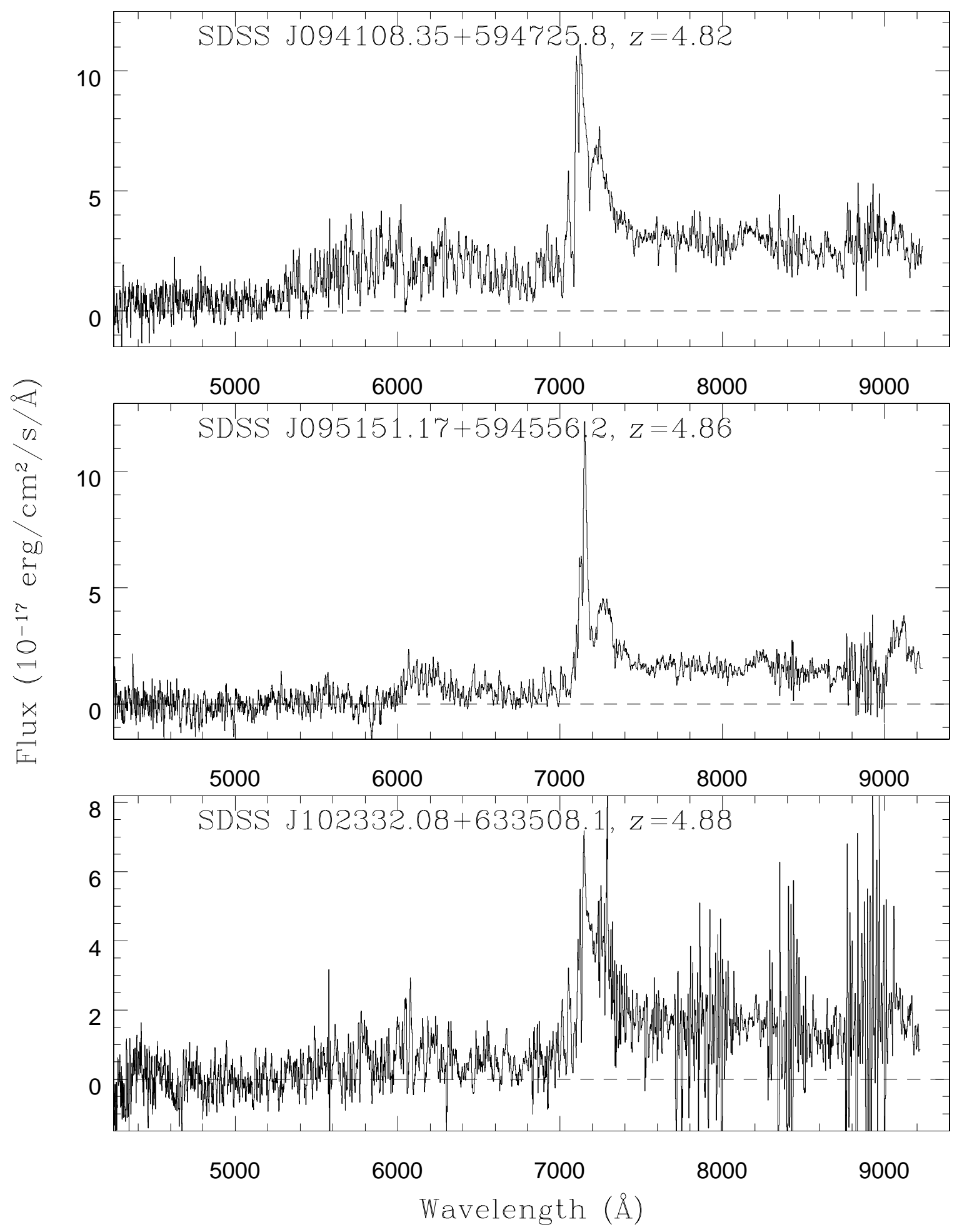




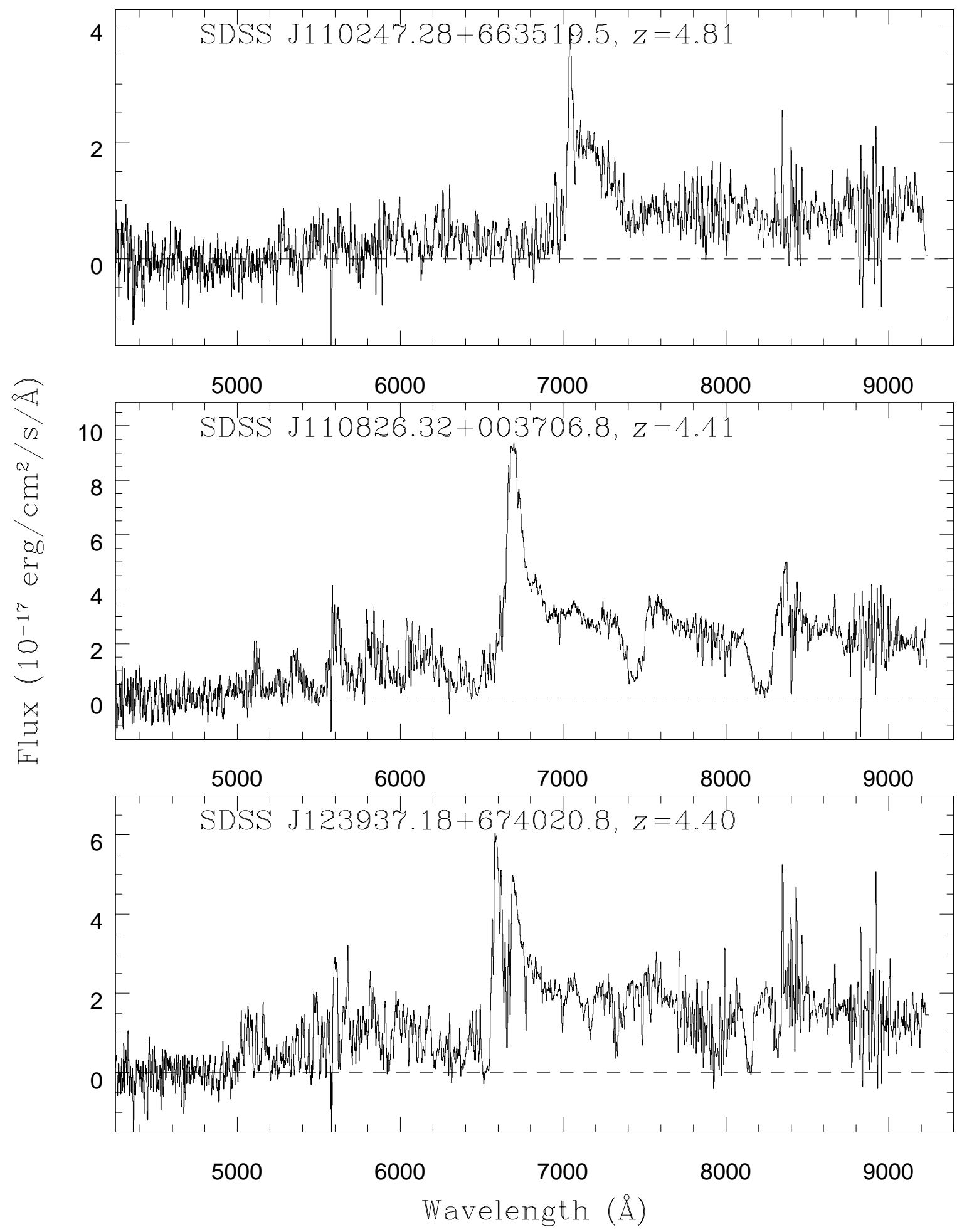




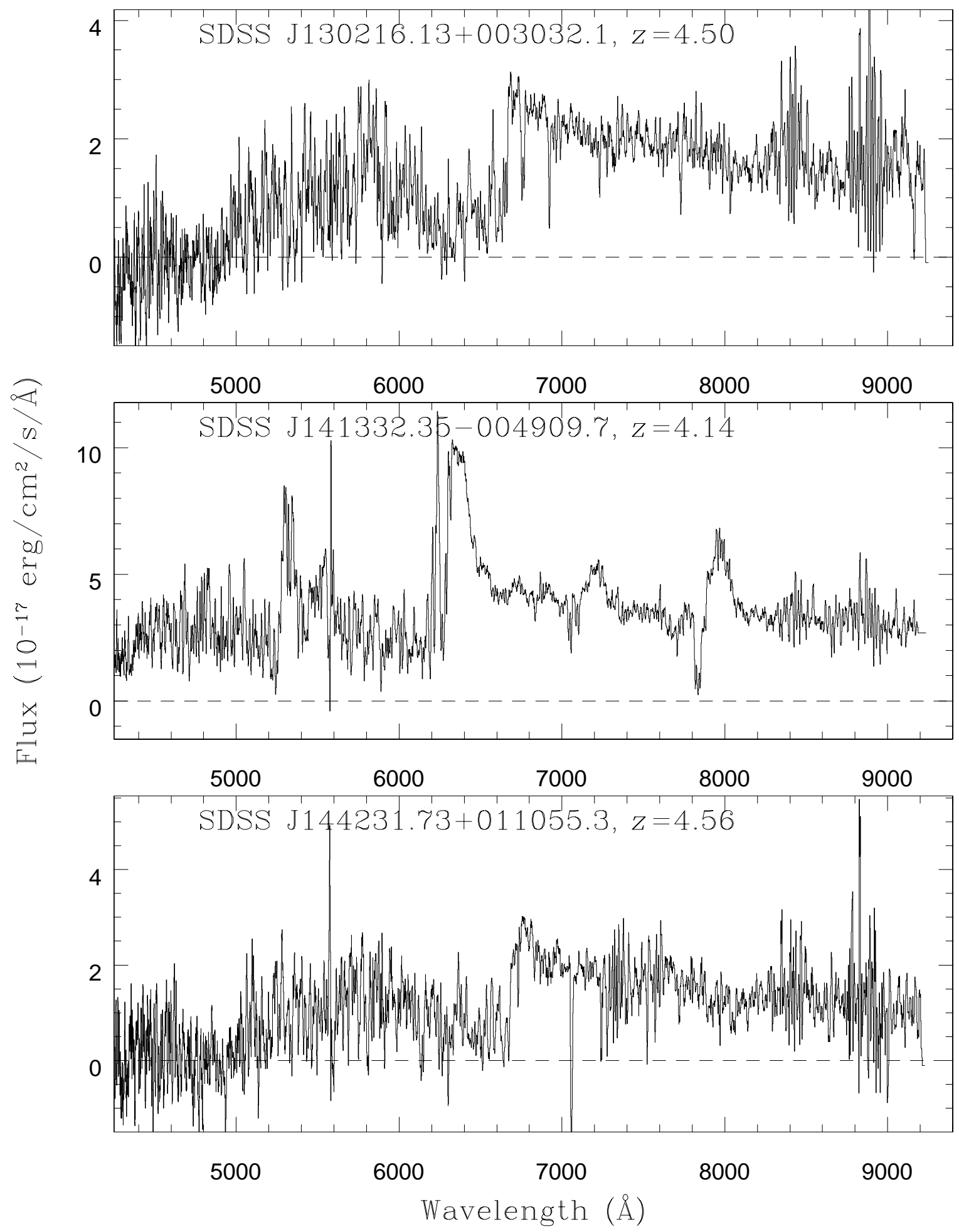




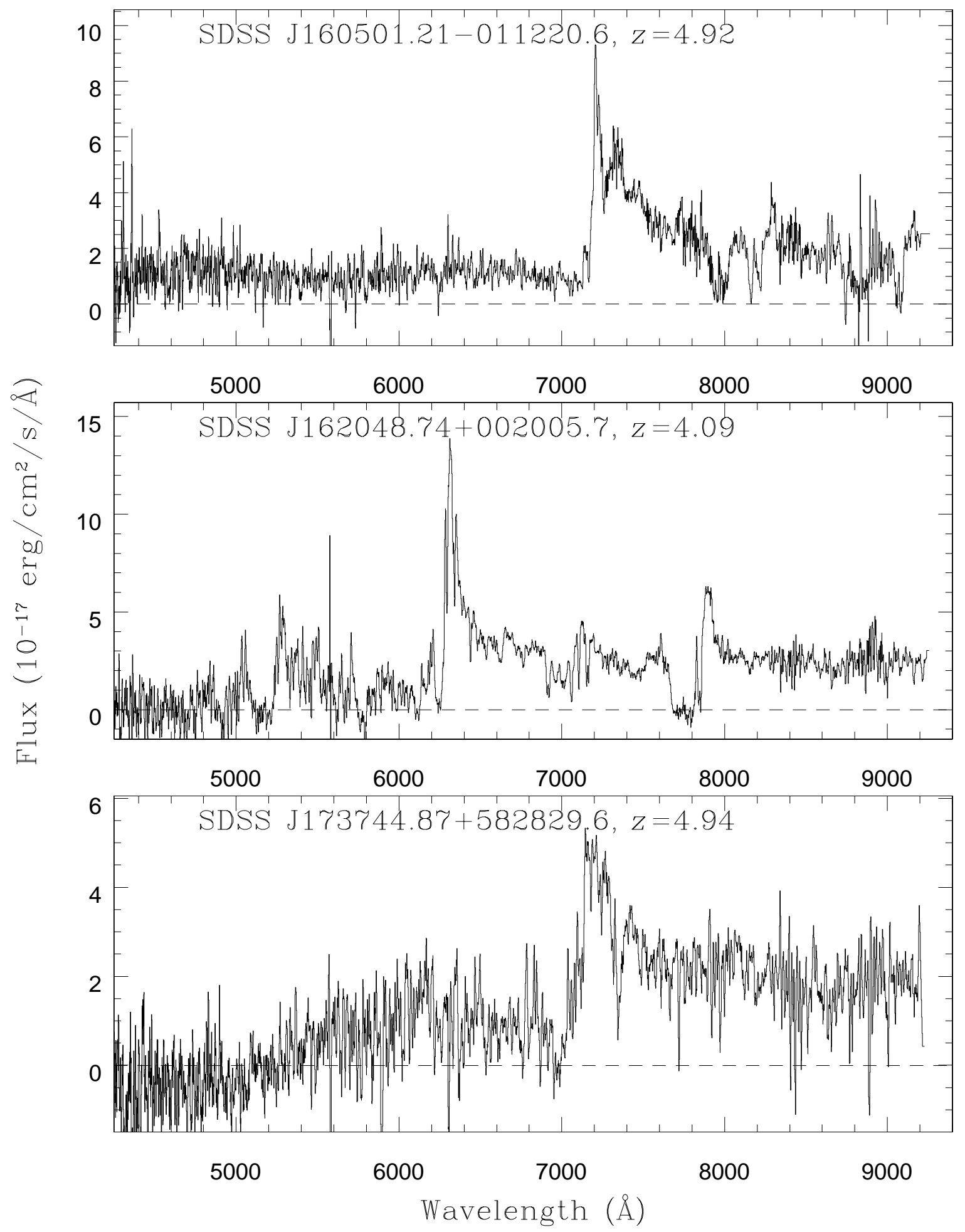



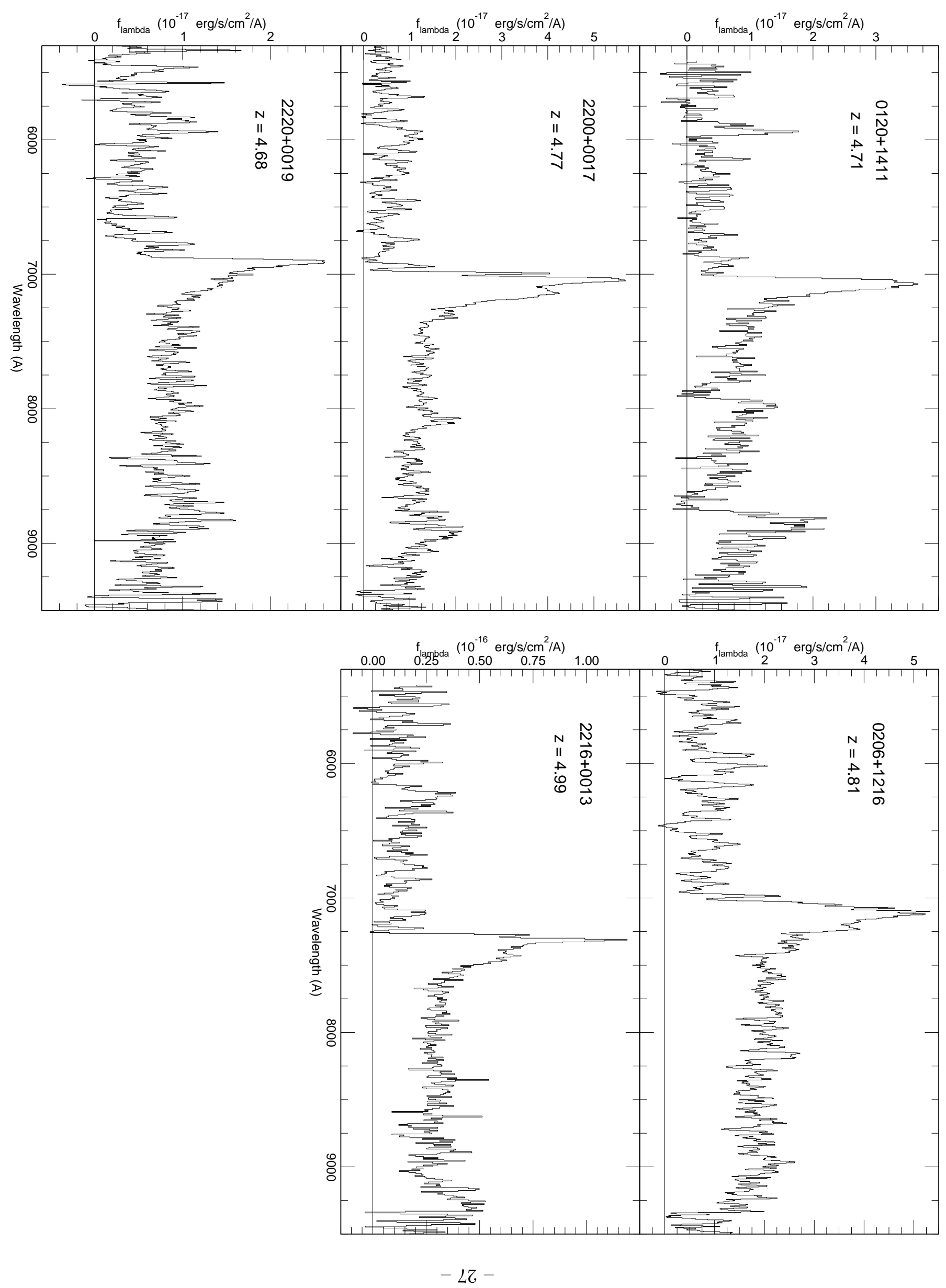
$-28-$
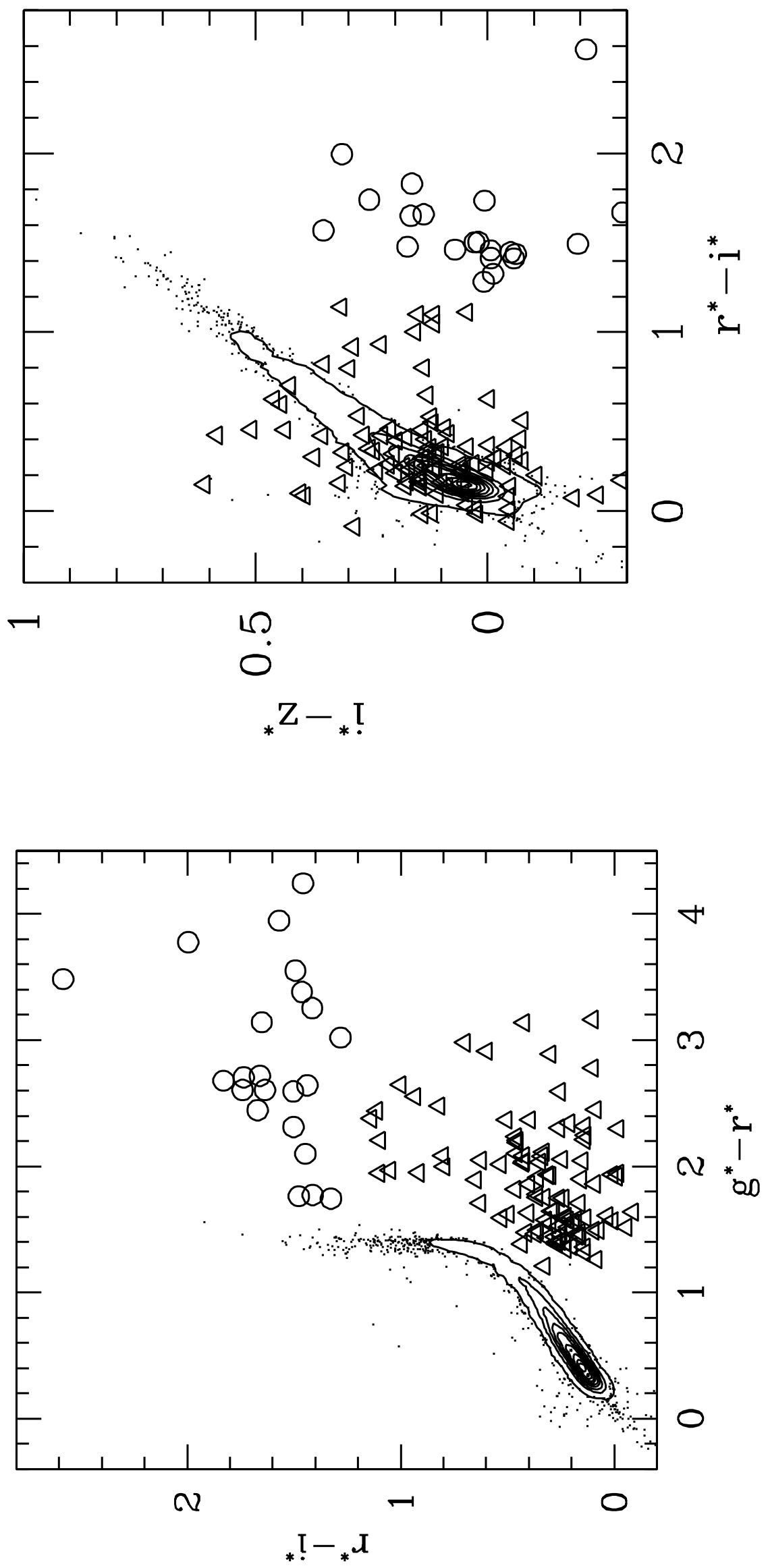


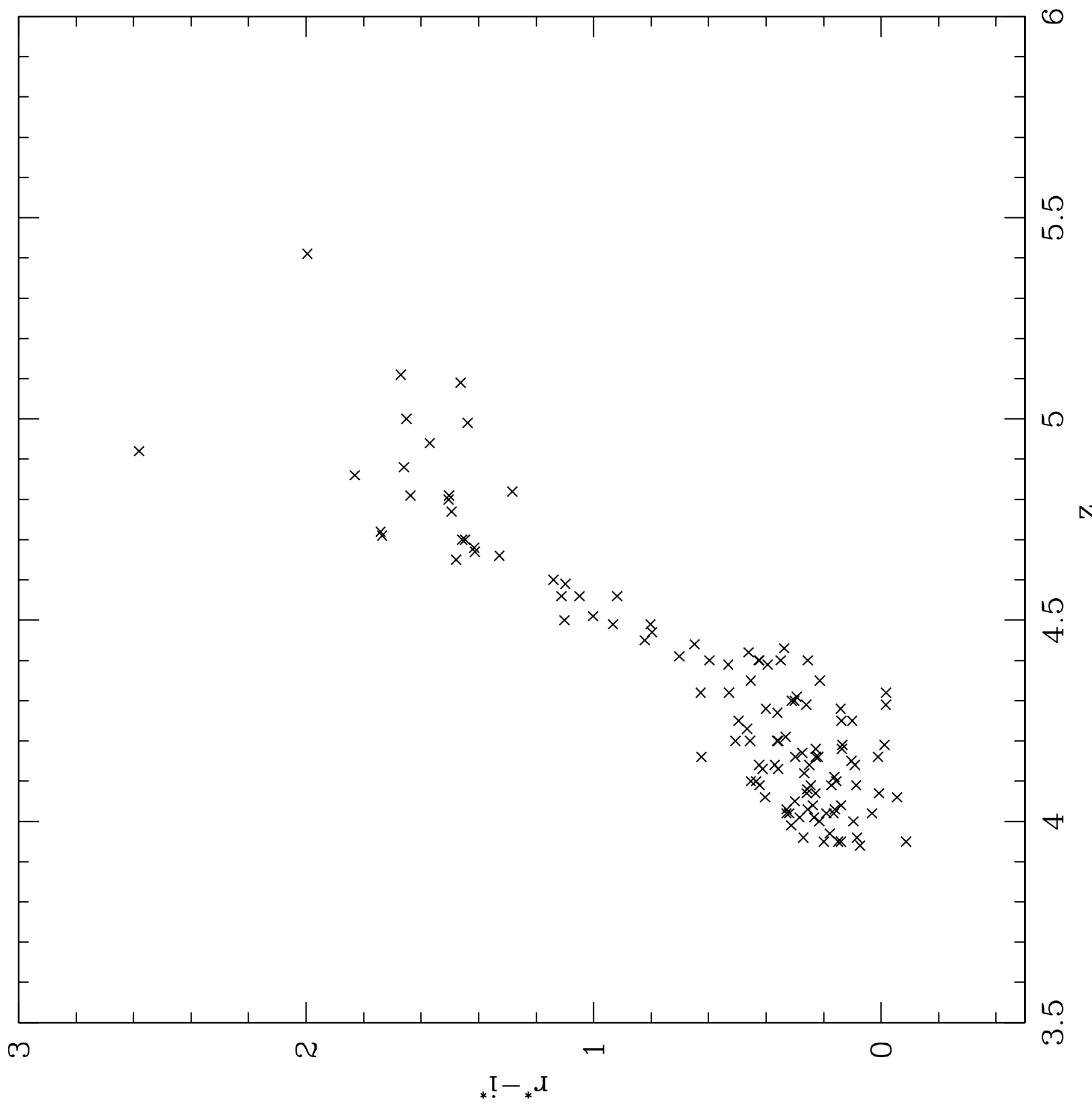




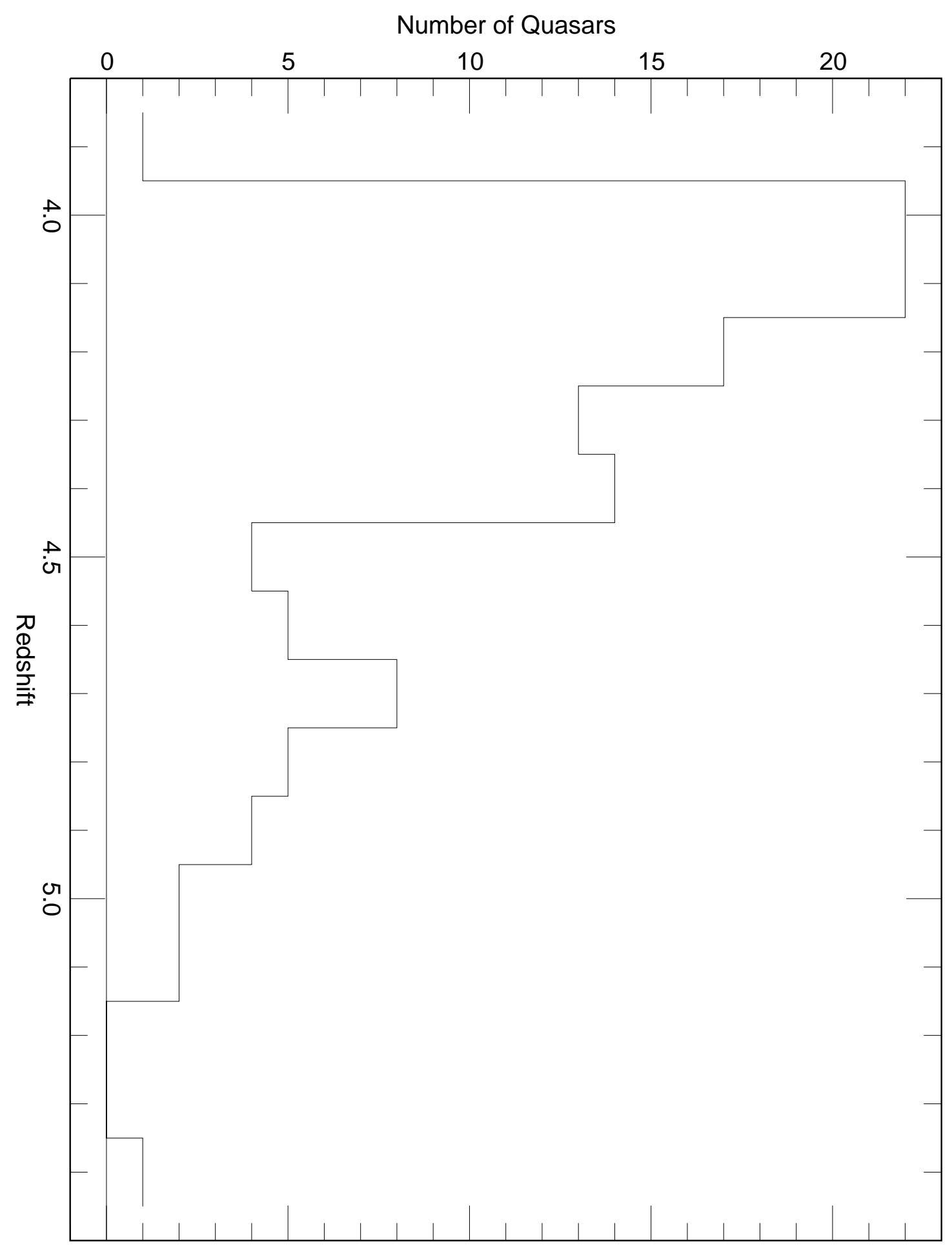




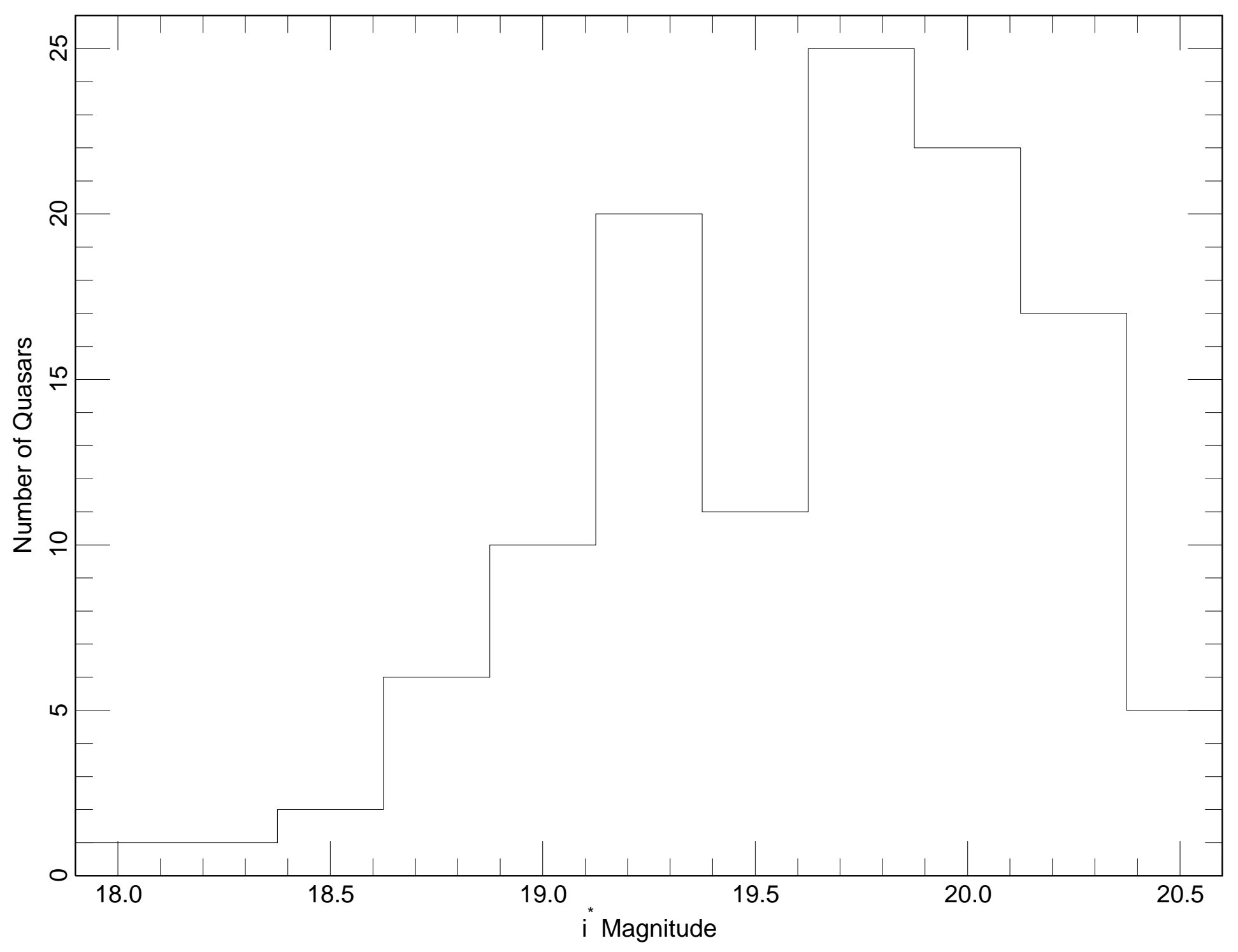


TABLE 1. Positions and Photometry of SDSS High-Redshift Quasars

\begin{tabular}{|c|c|c|c|c|c|c|c|}
\hline Quasar (SDSSp J) & Redshift & $u^{*}$ & $g^{*}$ & $r^{*}$ & $i^{*}$ & $z^{*}$ & Note \\
\hline $001950.05-004040.8$ & $4.31 \pm 0.01$ & $24.17 \pm 0.92$ & $21.49 \pm 0.07$ & $19.82 \pm 0.02$ & $19.50 \pm 0.02$ & $19.35 \pm 0.07$ & 8 \\
\hline $003126.80+150739.6$ & $4.20 \pm 0.01$ & $22.95 \pm 0.44$ & $22.32 \pm 0.11$ & $20.36 \pm 0.03$ & $19.97 \pm 0.03$ & $19.81 \pm 0.11$ & \\
\hline $003749.19+155208.4$ & $4.05 \pm 0.01$ & $23.79 \pm 0.56$ & $21.66 \pm 0.06$ & $20.06 \pm 0.02$ & $19.72 \pm 0.03$ & $19.71 \pm 0.10$ & \\
\hline $005006.35-005319.3$ & $4.32 \pm 0.01$ & $23.65 \pm 0.58$ & $21.93 \pm 0.09$ & $20.18 \pm 0.03$ & $19.53 \pm 0.02$ & $19.50 \pm 0.09$ & \\
\hline $005922.65+000301.4$ & $4.15 \pm 0.01$ & $23.68 \pm 0.78$ & $22.45 \pm 0.16$ & $19.26 \pm 0.01$ & $19.14 \pm 0.02$ & $19.07 \pm 0.07$ & 3 \\
\hline $010619.24+004823.4$ & $4.43 \pm 0.01$ & $23.78 \pm 0.60$ & $21.16 \pm 0.04$ & $19.05 \pm 0.01$ & $18.70 \pm 0.01$ & $18.44 \pm 0.03$ & 4 \\
\hline $012004.83+141108.3^{*}$ & $4.71 \pm 0.02$ & $23.48 \pm 0.51$ & $24.72 \pm 0.51$ & $21.98 \pm 0.10$ & $20.22 \pm 0.03$ & $20.19 \pm 0.11$ & 1 \\
\hline $012019.99+000735.6$ & $4.09 \pm 0.02$ & $23.77 \pm 0.82$ & $21.51 \pm 0.06$ & $20.02 \pm 0.03$ & $19.82 \pm 0.03$ & $20.08 \pm 0.16$ & 8 \\
\hline $015032.87+143425.6^{*}$ & $4.14 \pm 0.02$ & $24.21 \pm 0.69$ & $23.76 \pm 0.32$ & $20.56 \pm 0.04$ & $20.09 \pm 0.04$ & $19.47 \pm 0.08$ & \\
\hline $015339.61-001104.9$ & $4.19 \pm 0.01$ & $23.77 \pm 0.55$ & $21.17 \pm 0.04$ & $18.90 \pm 0.01$ & $18.74 \pm 0.01$ & $18.58 \pm 0.04$ & 4 \\
\hline $015642.11+141944.4^{*}$ & $4.30 \pm 0.02$ & $23.90 \pm 0.64$ & $23.61 \pm 0.28$ & $20.67 \pm 0.04$ & $20.33 \pm 0.04$ & $19.92 \pm 0.09$ & \\
\hline $015704.10+122858.3$ & $4.16 \pm 0.02$ & $23.84 \pm 0.66$ & $22.24 \pm 0.13$ & $20.24 \pm 0.03$ & $19.90 \pm 0.03$ & $19.72 \pm 0.09$ & \\
\hline $020651.37+121624.4$ & $4.81 \pm 0.02$ & $25.13 \pm 0.46$ & $24.05 \pm 0.46$ & $21.51 \pm 0.07$ & $19.86 \pm 0.03$ & $19.70 \pm 0.08$ & 1 \\
\hline $021043.15-001818.5$ & $4.70 \pm 0.02$ & $23.59 \pm 0.67$ & $22.87 \pm 0.16$ & $20.74 \pm 0.04$ & $19.28 \pm 0.02$ & $19.31 \pm 0.06$ & 8 \\
\hline $023137.65-072854.5$ & $5.41 \pm 0.02$ & $24.54 \pm 1.20$ & $25.36 \pm 0.63$ & $21.54 \pm 0.08$ & $19.53 \pm 0.02$ & $19.19 \pm 0.07$ & \\
\hline 023923.47-081005.1* & $4.00 \pm 0.02$ & $24.75 \pm 1.31$ & $20.90 \pm 0.03$ & $19.38 \pm 0.02$ & $19.27 \pm 0.02$ & $19.14 \pm 0.07$ & \\
\hline $024447.79-081606.1$ & $4.03 \pm 0.02$ & $24.20 \pm 1.48$ & $19.69 \pm 0.02$ & $18.21 \pm 0.02$ & $18.03 \pm 0.01$ & $17.92 \pm 0.03$ & \\
\hline 024457.19-010809.9* & $3.96 \pm 0.02$ & $22.81 \pm 0.38$ & $20.04 \pm 0.02$ & $18.62 \pm 0.01$ & $18.33 \pm 0.01$ & $18.16 \pm 0.02$ & 2 \\
\hline $025039.17-065405.1$ & $4.45 \pm 0.01$ & $25.00 \pm 1.23$ & $23.20 \pm 0.23$ & $20.68 \pm 0.04$ & $19.84 \pm 0.03$ & $19.46 \pm 0.09$ & \\
\hline $025159.41-084258.1$ & $4.25 \pm 0.01$ & $23.06 \pm 0.82$ & $23.52 \pm 0.32$ & $20.69 \pm 0.04$ & $20.56 \pm 0.05$ & $20.13 \pm 0.14$ & \\
\hline $025204.29+003136.9^{*}$ & $4.10 \pm 0.02$ & $24.88 \pm 0.66$ & $22.43 \pm 0.14$ & $20.15 \pm 0.03$ & $19.64 \pm 0.03$ & $19.15 \pm 0.06$ & \\
\hline $025518.58+004847.6$ & $3.99 \pm 0.01$ & $23.92 \pm 0.61$ & $20.59 \pm 0.02$ & $19.07 \pm 0.01$ & $18.70 \pm 0.01$ & $18.71 \pm 0.04$ & \\
\hline $025647.06-085041.4^{*}$ & $4.21 \pm 0.02$ & $24.08 \pm 0.73$ & $22.14 \pm 0.12$ & $19.98 \pm 0.02$ & $19.62 \pm 0.03$ & $19.46 \pm 0.08$ & \\
\hline $030025.23+003224.3$ & $4.19 \pm 0.01$ & $22.76 \pm 0.32$ & $22.01 \pm 0.09$ & $19.99 \pm 0.02$ & $19.94 \pm 0.03$ & $19.76 \pm 0.09$ & 8 \\
\hline $031213.98-062658.8$ & $4.03 \pm 0.01$ & $24.89 \pm 1.26$ & $20.71 \pm 0.03$ & $19.44 \pm 0.02$ & $19.08 \pm 0.02$ & $18.93 \pm 0.05$ & \\
\hline $032226.10-055824.7$ & $3.94 \pm 0.01$ & $25.87 \pm 0.85$ & $21.56 \pm 0.06$ & $20.01 \pm 0.03$ & $19.90 \pm 0.04$ & $20.06 \pm 0.14$ & \\
\hline $032608.12-003340.1$ & $4.18 \pm 0.01$ & $23.48 \pm 0.61$ & $21.17 \pm 0.04$ & $19.47 \pm 0.01$ & $19.26 \pm 0.02$ & $19.13 \pm 0.06$ & 6 \\
\hline $033119.67-074143.1$ & $4.70 \pm 0.01$ & $24.90 \pm 1.19$ & $24.93 \pm 0.84$ & $20.61 \pm 0.04$ & $19.10 \pm 0.02$ & $19.07 \pm 0.07$ & \\
\hline 033305.32-053709.0* & $4.09 \pm 0.01$ & $24.26 \pm 1.19$ & $22.40 \pm 0.10$ & $19.90 \pm 0.03$ & $19.79 \pm 0.04$ & $19.37 \pm 0.07$ & \\
\hline $033344.43-060625.2$ & $4.09 \pm 0.01$ & $23.35 \pm 0.64$ & $22.80 \pm 0.15$ & $20.70 \pm 0.04$ & $20.43 \pm 0.05$ & $20.10 \pm 0.13$ & \\
\hline $033406.99-063406.5$ & $3.95 \pm 0.01$ & $25.55 \pm 0.89$ & $20.75 \pm 0.03$ & $19.35 \pm 0.02$ & $19.18 \pm 0.02$ & $19.21 \pm 0.07$ & \\
\hline $033829.31+002156.3$ & $5.00 \pm 0.02$ & $23.70 \pm 0.61$ & $24.92 \pm 0.51$ & $21.68 \pm 0.09$ & $19.97 \pm 0.03$ & $19.75 \pm 0.13$ & 6 \\
\hline $034109.35-064805.1$ & $4.08 \pm 0.01$ & $25.32 \pm 0.88$ & $22.07 \pm 0.08$ & $20.49 \pm 0.03$ & $20.20 \pm 0.04$ & $20.20 \pm 0.15$ & \\
\hline $034541.51-072315.3$ & $4.02 \pm 0.02$ & $25.30 \pm 0.91$ & $21.73 \pm 0.07$ & $19.90 \pm 0.02$ & $19.52 \pm 0.02$ & $19.16 \pm 0.06$ & \\
\hline 034946.61-065730.3* & $3.95 \pm 0.02$ & $23.78 \pm 0.93$ & $22.59 \pm 0.13$ & $20.44 \pm 0.03$ & $20.23 \pm 0.03$ & $19.56 \pm 0.08$ & \\
\hline $073147.01+364346.5$ & $4.03 \pm 0.01$ & $24.48 \pm 0.98$ & $21.05 \pm 0.04$ & $19.58 \pm 0.03$ & $19.29 \pm 0.02$ & $19.20 \pm 0.06$ & \\
\hline $075103.96+424211.6$ & $4.16 \pm 0.01$ & $23.79 \pm 0.72$ & $20.69 \pm 0.03$ & $19.07 \pm 0.02$ & $18.82 \pm 0.02$ & $18.80 \pm 0.04$ & \\
\hline $075618.14+410408.6$ & $5.09 \pm 0.01$ & $24.53 \pm 0.91$ & $25.13 \pm 0.62$ & $21.70 \pm 0.08$ & $20.21 \pm 0.03$ & $20.11 \pm 0.12$ & \\
\hline $075652.07+450258.9$ & $4.80 \pm 0.01$ & $24.47 \pm 0.95$ & $24.41 \pm 0.49$ & $21.78 \pm 0.09$ & $20.24 \pm 0.04$ & $20.20 \pm 0.13$ & \\
\hline $075732.89+441424.7$ & $4.16 \pm 0.02$ & $23.70 \pm 0.48$ & $21.02 \pm 0.03$ & $19.63 \pm 0.02$ & $19.38 \pm 0.02$ & $19.30 \pm 0.06$ & \\
\hline $080159.25+433625.0$ & $4.17 \pm 0.01$ & $24.07 \pm 0.80$ & $22.04 \pm 0.08$ & $20.56 \pm 0.03$ & $20.26 \pm 0.03$ & $20.24 \pm 0.10$ & \\
\hline $080549.94+482345.9$ & $4.18 \pm 0.03$ & $25.63 \pm 0.74$ & $21.76 \pm 0.07$ & $20.11 \pm 0.03$ & $19.86 \pm 0.03$ & $19.59 \pm 0.07$ & \\
\hline $081054.88+460357.9$ & $4.07 \pm 0.01$ & $25.45 \pm 0.88$ & $20.46 \pm 0.03$ & $18.67 \pm 0.01$ & $18.38 \pm 0.02$ & $18.30 \pm 0.03$ & \\
\hline $081241.12+442129.0$ & $4.32 \pm 0.01$ & $22.91 \pm 0.50$ & $21.98 \pm 0.08$ & $19.97 \pm 0.03$ & $19.95 \pm 0.04$ & $19.77 \pm 0.08$ & \\
\hline $083046.28+474646.5$ & $4.29 \pm 0.01$ & $25.40 \pm 0.73$ & $23.03 \pm 0.22$ & $20.69 \pm 0.04$ & $20.40 \pm 0.04$ & $20.16 \pm 0.11$ & \\
\hline
\end{tabular}


TABLE 1. Positions and Photometry of SDSS High-Redshift Quasars (continued)

\begin{tabular}{|c|c|c|c|c|c|c|c|}
\hline Quasar (SDSSp J) & Redshift & $u^{*}$ & $g^{*}$ & $r^{*}$ & $i^{*}$ & $z^{*}$ & Note \\
\hline $083103.00+523533.6$ & $4.44 \pm 0.01$ & $23.83 \pm 0.89$ & $22.00 \pm 0.08$ & $20.05 \pm 0.02$ & $19.37 \pm 0.02$ & $19.22 \pm 0.07$ & \\
\hline $083212.37+530327.4$ & $4.02 \pm 0.01$ & $23.60 \pm 0.74$ & $21.44 \pm 0.05$ & $19.82 \pm 0.03$ & $19.46 \pm 0.03$ & $19.23 \pm 0.06$ & \\
\hline $083324.57+523955.0$ & $3.97 \pm 0.01$ & $25.03 \pm 0.91$ & $21.76 \pm 0.06$ & $20.14 \pm 0.03$ & $19.94 \pm 0.03$ & $19.77 \pm 0.09$ & \\
\hline $083946.22+511202.8$ & $4.39 \pm 0.01$ & $25.22 \pm 0.78$ & $21.78 \pm 0.06$ & $19.39 \pm 0.02$ & $18.98 \pm 0.02$ & $18.77 \pm 0.04$ & \\
\hline $084811.52-001418.0$ & $4.12 \pm 0.01$ & $23.27 \pm 0.42$ & $20.71 \pm 0.03$ & $19.28 \pm 0.01$ & $18.99 \pm 0.01$ & $18.94 \pm 0.04$ & \\
\hline $085151.27+020756.1$ & $4.28 \pm 0.01$ & $24.22 \pm 0.96$ & $21.70 \pm 0.06$ & $19.45 \pm 0.02$ & $19.29 \pm 0.02$ & $19.09 \pm 0.06$ & \\
\hline $085210.89+535949.0^{*}$ & $4.20 \pm 0.02$ & $23.75 \pm 0.63$ & $22.42 \pm 0.10$ & $20.29 \pm 0.03$ & $19.82 \pm 0.03$ & $19.29 \pm 0.05$ & \\
\hline $085430.18+004213.6$ & $4.07 \pm 0.01$ & $24.25 \pm 0.73$ & $21.91 \pm 0.07$ & $20.28 \pm 0.03$ & $20.02 \pm 0.04$ & $19.82 \pm 0.09$ & \\
\hline $085634.93+525206.4^{*}$ & $4.79 \pm 0.10$ & $25.16 \pm 0.92$ & $24.69 \pm 0.50$ & $22.06 \pm 0.11$ & $20.31 \pm 0.04$ & $20.04 \pm 0.09$ & \\
\hline $090242.08-002125.9$ & $4.39 \pm 0.02$ & $25.43 \pm 0.68$ & $22.38 \pm 0.09$ & $20.33 \pm 0.03$ & $19.77 \pm 0.02$ & $19.47 \pm 0.06$ & \\
\hline $090440.64+535038.8$ & $4.25 \pm 0.02$ & $23.83 \pm 0.77$ & $21.40 \pm 0.04$ & $19.76 \pm 0.02$ & $19.25 \pm 0.04$ & $19.13 \pm 0.04$ & \\
\hline $090532.15-001430.5$ & $4.25 \pm 0.01$ & $25.01 \pm 0.77$ & $22.42 \pm 0.10$ & $20.05 \pm 0.03$ & $19.89 \pm 0.02$ & $19.77 \pm 0.08$ & \\
\hline $091016.79+575331.1$ & $4.01 \pm 0.03$ & $24.73 \pm 1.31$ & $21.51 \pm 0.05$ & $19.81 \pm 0.03$ & $19.50 \pm 0.03$ & $19.55 \pm 0.07$ & \\
\hline $091316.56+591921.5$ & $5.11 \pm 0.02$ & $24.24 \pm 1.06$ & $24.68 \pm 0.55$ & $22.19 \pm 0.14$ & $20.50 \pm 0.05$ & $20.76 \pm 0.25$ & \\
\hline $092038.49+564235.9$ & $4.14 \pm 0.01$ & $24.47 \pm 1.01$ & $21.85 \pm 0.06$ & $20.34 \pm 0.03$ & $19.95 \pm 0.03$ & $19.92 \pm 0.12$ & \\
\hline $092256.20+561849.3$ & $4.19 \pm 0.02$ & $23.83 \pm 0.90$ & $21.17 \pm 0.04$ & $19.37 \pm 0.03$ & $18.99 \pm 0.02$ & $18.92 \pm 0.04$ & \\
\hline $094056.02+584830.2$ & $4.66 \pm 0.01$ & $24.19 \pm 0.96$ & $22.37 \pm 0.10$ & $20.61 \pm 0.04$ & $19.27 \pm 0.02$ & $19.27 \pm 0.07$ & \\
\hline $094108.36+594725.8$ & $4.82 \pm 0.02$ & $23.91 \pm 0.77$ & $23.70 \pm 0.33$ & $20.66 \pm 0.04$ & $19.36 \pm 0.03$ & $19.34 \pm 0.06$ & \\
\hline $094917.17+602104.5$ & $4.28 \pm 0.01$ & $24.07 \pm 0.89$ & $22.55 \pm 0.14$ & $20.67 \pm 0.04$ & $20.26 \pm 0.04$ & $20.12 \pm 0.11$ & \\
\hline $095000.17+620318.6$ & $4.06 \pm 0.01$ & $25.81 \pm 0.67$ & $21.73 \pm 0.07$ & $20.19 \pm 0.03$ & $20.22 \pm 0.04$ & $20.25 \pm 0.17$ & \\
\hline $095151.17+594556.2$ & $4.86 \pm 0.03$ & $23.53 \pm 0.71$ & $24.34 \pm 0.48$ & $21.65 \pm 0.08$ & $19.81 \pm 0.03$ & $19.64 \pm 0.08$ & \\
\hline $100154.87+630818.0$ & $4.14 \pm 0.01$ & $25.09 \pm 0.96$ & $22.31 \pm 0.09$ & $20.43 \pm 0.03$ & $20.32 \pm 0.04$ & $20.54 \pm 0.18$ & \\
\hline $100413.14+630437.4$ & $4.11 \pm 0.02$ & $25.54 \pm 0.77$ & $22.15 \pm 0.10$ & $20.42 \pm 0.03$ & $20.24 \pm 0.04$ & $20.08 \pm 0.14$ & \\
\hline $101053.52+644832.0$ & $4.65 \pm 0.04$ & $23.90 \pm 0.80$ & $23.17 \pm 0.21$ & $21.37 \pm 0.06$ & $19.87 \pm 0.03$ & $19.67 \pm 0.08$ & \\
\hline $101549.00+002020.0$ & $4.40 \pm 0.02$ & $23.83 \pm 0.50$ & $21.78 \pm 0.06$ & $19.72 \pm 0.01$ & $19.27 \pm 0.01$ & $18.98 \pm 0.04$ & 5 \\
\hline $102043.82+000105.8$ & $4.16 \pm 0.04$ & $23.15 \pm 0.54$ & $22.57 \pm 0.15$ & $20.48 \pm 0.03$ & $19.83 \pm 0.02$ & $19.35 \pm 0.08$ & \\
\hline $102332.08+633508.1$ & $4.88 \pm 0.01$ & $23.07 \pm 0.48$ & $24.09 \pm 0.47$ & $21.36 \pm 0.06$ & $19.69 \pm 0.03$ & $19.55 \pm 0.07$ & \\
\hline $103309.21+644351.1$ & $3.95 \pm 0.02$ & $24.16 \pm 1.04$ & $21.47 \pm 0.06$ & $20.09 \pm 0.03$ & $19.89 \pm 0.03$ & $19.98 \pm 0.13$ & \\
\hline $104008.10+651429.3$ & $4.49 \pm 0.02$ & $24.05 \pm 0.97$ & $23.34 \pm 0.24$ & $20.77 \pm 0.04$ & $19.83 \pm 0.03$ & $19.59 \pm 0.08$ & \\
\hline $104040.14-001540.9$ & $4.32 \pm 0.03$ & $23.49 \pm 0.61$ & $21.00 \pm 0.04$ & $19.36 \pm 0.01$ & $18.80 \pm 0.01$ & $18.65 \pm 0.03$ & \\
\hline $104351.20+650647.7$ & $4.49 \pm 0.02$ & $26.30 \pm 0.55$ & $22.00 \pm 0.08$ & $19.90 \pm 0.03$ & $19.09 \pm 0.02$ & $18.95 \pm 0.05$ & \\
\hline $104837.40-002813.7$ & $4.00 \pm 0.02$ & $24.71 \pm 0.58$ & $20.86 \pm 0.03$ & $19.29 \pm 0.02$ & $19.05 \pm 0.07$ & $18.96 \pm 0.12$ & 9 \\
\hline $105254.60-000625.9$ & $4.13 \pm 0.01$ & $23.95 \pm 0.46$ & $21.47 \pm 0.04$ & $19.93 \pm 0.02$ & $19.54 \pm 0.02$ & $19.55 \pm 0.06$ & \\
\hline 105320.43-001649.6 & $4.29 \pm 0.01$ & $23.69 \pm 0.58$ & $21.70 \pm 0.07$ & $19.35 \pm 0.01$ & $19.33 \pm 0.02$ & $19.27 \pm 0.06$ & 5 \\
\hline $105602.37+003222.0$ & $4.02 \pm 0.01$ & $22.99 \pm 0.47$ & $21.47 \pm 0.05$ & $19.88 \pm 0.02$ & $19.66 \pm 0.02$ & $19.49 \pm 0.06$ & \\
\hline $105902.73+010404.1$ & $4.06 \pm 0.01$ & $23.69 \pm 0.36$ & $21.30 \pm 0.04$ & $19.64 \pm 0.01$ & $19.21 \pm 0.01$ & $19.26 \pm 0.05$ & \\
\hline $110247.29+663519.5$ & $4.81 \pm 0.03$ & $24.55 \pm 1.18$ & $24.74 \pm 0.53$ & $22.12 \pm 0.12$ & $20.47 \pm 0.04$ & $20.98 \pm 0.32$ & \\
\hline $110813.86-005944.5$ & $4.01 \pm 0.01$ & $24.20 \pm 0.48$ & $20.96 \pm 0.03$ & $19.51 \pm 0.01$ & $19.25 \pm 0.02$ & $19.11 \pm 0.05$ & 9 \\
\hline $110819.16-005824.0$ & $4.56 \pm 0.02$ & $23.97 \pm 0.48$ & $22.96 \pm 0.18$ & $20.94 \pm 0.04$ & $19.87 \pm 0.02$ & $19.72 \pm 0.08$ & \\
\hline $110826.32+003706.8^{*}$ & $4.41 \pm 0.02$ & $24.36 \pm 0.73$ & $23.58 \pm 0.33$ & $20.57 \pm 0.03$ & $19.84 \pm 0.03$ & $19.40 \pm 0.06$ & \\
\hline $111224.18+004630.4$ & $4.02 \pm 0.01$ & $24.32 \pm 0.46$ & $21.36 \pm 0.04$ & $19.71 \pm 0.01$ & $19.66 \pm 0.02$ & $19.59 \pm 0.07$ & \\
\hline $111401.47-005321.2$ & $4.59 \pm 0.01$ & $23.43 \pm 0.44$ & $22.95 \pm 0.19$ & $20.70 \pm 0.03$ & $19.57 \pm 0.02$ & $19.44 \pm 0.06$ & 7 \\
\hline $112253.50+005329.8$ & $4.56 \pm 0.02$ & $23.28 \pm 0.59$ & $22.73 \pm 0.19$ & $20.26 \pm 0.03$ & $19.12 \pm 0.02$ & $19.05 \pm 0.05$ & 7 \\
\hline $113559.94+002422.8$ & $4.04 \pm 0.02$ & $23.60 \pm 0.37$ & $21.35 \pm 0.04$ & $20.02 \pm 0.02$ & $19.87 \pm 0.02$ & $19.71 \pm 0.08$ & 9 \\
\hline $123937.18+674020.8^{*}$ & $4.40 \pm 0.02$ & $25.83 \pm 0.66$ & $23.71 \pm 0.29$ & $20.79 \pm 0.04$ & $20.18 \pm 0.04$ & $19.72 \pm 0.11$ & \\
\hline
\end{tabular}


TABLE 1. Positions and Photometry of SDSS High-Redshift Quasars (continued)

\begin{tabular}{|c|c|c|c|c|c|c|c|}
\hline Quasar (SDSSp J) & Redshift & $u^{*}$ & $g^{*}$ & $r^{*}$ & $i^{*}$ & $z^{*}$ & Note \\
\hline $125433.57-003922.7$ & $4.23 \pm 0.03$ & $23.50 \pm 0.53$ & $22.42 \pm 0.10$ & $20.58 \pm 0.03$ & $20.10 \pm 0.03$ & $19.99 \pm 0.08$ & \\
\hline $125759.22-011130.3$ & $4.10 \pm 0.01$ & $24.39 \pm 0.53$ & $20.40 \pm 0.02$ & $19.00 \pm 0.01$ & $18.55 \pm 0.01$ & $18.45 \pm 0.03$ & \\
\hline $130216.13+003032.1$ & $4.51 \pm 0.04$ & $23.70 \pm 0.52$ & $22.95 \pm 0.18$ & $20.98 \pm 0.04$ & $19.86 \pm 0.02$ & $19.69 \pm 0.08$ & \\
\hline $131052.51-005533.2$ & $4.16 \pm 0.01$ & $23.19 \pm 0.81$ & $20.82 \pm 0.03$ & $18.86 \pm 0.01$ & $18.83 \pm 0.01$ & $18.79 \pm 0.04$ & 7 \\
\hline $132110.82+003821.7$ & $4.67 \pm 0.02$ & $23.94 \pm 0.62$ & $23.28 \pm 0.20$ & $21.48 \pm 0.07$ & $20.04 \pm 0.03$ & $20.08 \pm 0.10$ & 7 \\
\hline $132447.26-031358.3$ & $4.02 \pm 0.01$ & $23.12 \pm 0.47$ & $21.50 \pm 0.06$ & $19.98 \pm 0.02$ & $19.79 \pm 0.03$ & $19.60 \pm 0.15$ & \\
\hline $134723.09+002158.9$ & $4.27 \pm 0.01$ & $23.59 \pm 0.55$ & $21.15 \pm 0.03$ & $19.34 \pm 0.01$ & $18.96 \pm 0.01$ & $18.85 \pm 0.04$ & \\
\hline $135057.86-004355.3$ & $4.40 \pm 0.01$ & $24.03 \pm 0.55$ & $22.36 \pm 0.09$ & $20.29 \pm 0.02$ & $19.91 \pm 0.02$ & $19.64 \pm 0.06$ & \\
\hline $135134.46-003652.2$ & $4.04 \pm 0.01$ & $23.16 \pm 0.45$ & $21.92 \pm 0.06$ & $20.14 \pm 0.02$ & $19.88 \pm 0.03$ & $19.75 \pm 0.08$ & \\
\hline 135423.00-003906.2 & $4.42 \pm 0.01$ & $24.19 \pm 0.56$ & $22.92 \pm 0.14$ & $20.64 \pm 0.03$ & $20.15 \pm 0.03$ & $19.92 \pm 0.07$ & \\
\hline $141315.36+000032.4$ & $4.07 \pm 0.01$ & $23.68 \pm 0.57$ & $21.38 \pm 0.05$ & $19.76 \pm 0.02$ & $19.73 \pm 0.02$ & $19.75 \pm 0.10$ & 7 \\
\hline $141332.36-004909.7^{*}$ & $4.14 \pm 0.02$ & $25.35 \pm 0.93$ & $21.09 \pm 0.04$ & $19.58 \pm 0.01$ & $19.30 \pm 0.02$ & $19.09 \pm 0.05$ & 7 \\
\hline $144231.73+011055.3$ & $4.56 \pm 0.03$ & $24.00 \pm 0.39$ & $22.86 \pm 0.13$ & $20.87 \pm 0.04$ & $19.93 \pm 0.03$ & $19.62 \pm 0.08$ & \\
\hline $144407.63-010152.8$ & $4.51 \pm 0.01$ & $24.41 \pm 0.52$ & $23.01 \pm 0.23$ & $20.32 \pm 0.03$ & $19.29 \pm 0.02$ & $19.11 \pm 0.05$ & \\
\hline $144617.35-010131.2$ & $4.13 \pm 0.01$ & $24.69 \pm 0.44$ & $21.06 \pm 0.04$ & $19.53 \pm 0.01$ & $19.09 \pm 0.01$ & $18.89 \pm 0.04$ & \\
\hline $152443.19+011358.9$ & $4.10 \pm 0.02$ & $23.54 \pm 0.44$ & $22.12 \pm 0.08$ & $20.17 \pm 0.02$ & $19.98 \pm 0.03$ & $19.62 \pm 0.09$ & \\
\hline $152740.52-010602.7$ & $4.40 \pm 0.01$ & $24.15 \pm 0.53$ & $22.73 \pm 0.15$ & $20.48 \pm 0.03$ & $19.95 \pm 0.02$ & $19.63 \pm 0.07$ & 7 \\
\hline $160501.21-011220.6^{*}$ & $4.92 \pm 0.02$ & $24.50 \pm 0.48$ & $26.19 \pm 0.38$ & $22.50 \pm 0.15$ & $19.78 \pm 0.02$ & $19.87 \pm 0.08$ & 7 \\
\hline $162048.74+002005.7^{*}$ & $4.09 \pm 0.02$ & $24.30 \pm 0.41$ & $21.99 \pm 0.06$ & $19.85 \pm 0.02$ & $19.36 \pm 0.02$ & $18.95 \pm 0.05$ & \\
\hline $170804.91+602202.0$ & $4.35 \pm 0.02$ & $23.18 \pm 0.75$ & $22.47 \pm 0.10$ & $20.24 \pm 0.02$ & $19.78 \pm 0.03$ & $19.62 \pm 0.09$ & \\
\hline $171014.52+592326.5$ & $4.47 \pm 0.03$ & $23.03 \pm 0.42$ & $22.50 \pm 0.11$ & $20.48 \pm 0.03$ & $19.67 \pm 0.02$ & $19.36 \pm 0.07$ & \\
\hline $171224.92+560625.0$ & $4.20 \pm 0.01$ & $23.72 \pm 0.62$ & $22.93 \pm 0.19$ & $20.54 \pm 0.03$ & $20.02 \pm 0.04$ & $20.08 \pm 0.11$ & \\
\hline $171530.49+645319.3$ & $3.96 \pm 0.01$ & $22.64 \pm 0.54$ & $20.85 \pm 0.04$ & $19.56 \pm 0.02$ & $19.46 \pm 0.02$ & $19.49 \pm 0.11$ & \\
\hline $171808.67+551511.2$ & $4.60 \pm 0.01$ & $24.24 \pm 0.50$ & $23.56 \pm 0.25$ & $21.15 \pm 0.05$ & $19.99 \pm 0.03$ & $19.65 \pm 0.08$ & \\
\hline $172007.20+602823.9$ & $4.40 \pm 0.01$ & $23.24 \pm 1.21$ & $23.09 \pm 0.21$ & $20.47 \pm 0.04$ & $20.20 \pm 0.04$ & $20.06 \pm 0.12$ & \\
\hline $173744.87+582829.5$ & $4.94 \pm 0.05$ & $24.69 \pm 0.31$ & $24.92 \pm 0.44$ & $20.93 \pm 0.05$ & $19.33 \pm 0.03$ & $18.95 \pm 0.07$ & \\
\hline $220008.66+001744.8$ & $4.77 \pm 0.01$ & $25.20 \pm 0.35$ & $24.28 \pm 0.38$ & $20.68 \pm 0.03$ & $19.15 \pm 0.01$ & $19.31 \pm 0.06$ & 1 \\
\hline $221644.02+001348.3$ & $4.99 \pm 0.01$ & $24.02 \pm 0.59$ & $24.49 \pm 0.46$ & $21.78 \pm 0.07$ & $20.30 \pm 0.04$ & $20.33 \pm 0.15$ & 1 \\
\hline $222050.80+001959.1$ & $4.68 \pm 0.02$ & $24.71 \pm 0.52$ & $24.99 \pm 0.57$ & $21.67 \pm 0.07$ & $20.21 \pm 0.03$ & $20.17 \pm 0.16$ & 1 \\
\hline $234147.27+001551.9$ & $3.95 \pm 0.01$ & $24.16 \pm 0.71$ & $21.63 \pm 0.06$ & $19.96 \pm 0.02$ & $20.03 \pm 0.04$ & $19.73 \pm 0.13$ & \\
\hline $235718.36+004350.4$ & $4.35 \pm 0.01$ & $24.00 \pm 0.78$ & $22.44 \pm 0.14$ & $20.07 \pm 0.03$ & $19.83 \pm 0.04$ & $19.61 \pm 0.10$ & 6 \\
\hline
\end{tabular}

Notes: Positions are in J2000.0 coordinates. An asterisk following an object name indicates a probable BAL quasar. Photometry is reported in terms of asinh magnitudes; see Lupton, Gunn, \& Szalay (1999) for details. In this system, zero flux corresponds to $24.6,25.1,24.8,24.4$, and 22.8 in $u^{*}, g^{*}, r^{*}, i^{*}$, and $z^{*}$, respectively.

1) Spectra obtained with APO 3.5-m

2) Unpublished PSS Quasar; see http://astro.caltech.edu/ george/z4.qsos

3) PSS Quasar; Kennefick et al. (1995a)

4) BRI Quasar; Finding Chart in Smith et al. (1994b)

5) BRI Quasar; Finding Chart in Smith et al. (1994a)

6) SDSS Quasar; Fan et al. (1999)

7) SDSS Quasar; Fan et al. (2000a)

8) SDSS Quasar; Fan et al. (2001)

9) SDSS Quasar; Schneider et al. (2001) 